\title{
MHD Modelling of Liquid Metal Films for Fusion Divertor Surface Protection
}

\section{RECEIVED \\ JUL 081997 \\ O S.TI}

Prospectus of Neil B. Morley

Mechanical, Aerospace and Nuclear Engineering

UNIVERSITY OF CALIFORNIA

Los Angeles

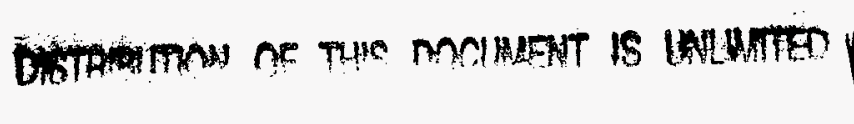
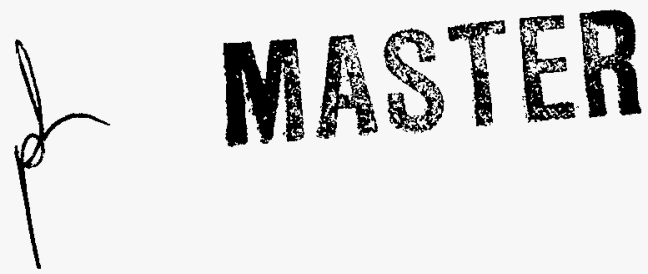

1991 


\section{DISCLAIMER}

This report was prepared as an account of work sponsored by an agency of the United States Government. Neither the United States Government nor any agency thereof, nor any of their employees, makes any warranty, express or implied, or assumes any legal liability or responsibility for the accuracy, completeness, or usefulness of any information, apparatus, product, or process disclosed, or represents that its use would not infringe privately owned rights. Reference herein to any specific commercial product, process, or service by trade name, trademark, manufacturer, or otherwise does not necessarily constitute or imply its endorsement, recommendation, or favoring by the United States Government or any agency thereof. The views and opinions of authors expressed herein do not necessarily state or reflect those of the United States Government or any agency thereof. 


\section{Contents}

List of Tables $\quad$ iv

List of Figures $\quad$ v

Abstract vi vi vis

1 Introduction 1

1.1 Liquid metal divertor system issues .............. 2

1.2 Goal of the research ................... 4

2 Review 7

2.1 Introduction to $\mathrm{LM}$ contact devises .............. 8

2.1.1 Choice of liquid metal .............. 8

2.1.2 Designs of LM plasma contact systems . . . . . . . . 11

2.2 Mathematical models of MHD film flow . . . . . . . . . . 19

3 Proposed mathematical models 26

3.1 Remaining questions .................. 27

3.2 Model-1: fully developed film .............. 31

3.2.1 Zero particle flux ................ 32 
3.2 .2 Non-zero particle flux . . . . . . . . . . 38

3.3 Model-2: developing film . . . . . . . . . . . . . 42

4 Experiment $\quad 44$

4.1 Construction of experimental facility . . . . . . . . 44

4.1 .1 Magnetic field system . . . . . . . . . . 45

4.1.2 LM circulation system $\ldots \ldots \ldots \ldots \ldots \ldots$

4.2 MHD-FFX design and test plan . . . . . . . . . 53

5 Summary $\quad 57$

$\begin{array}{ll}\text { Bibliography } & 60\end{array}$

A List of symbols and abbreviations $\quad 66$ 


\section{List of Tables}

1 SOL plasma parameters and resulting momentum flux $\ldots \ldots$

2 Magnet coil physical specifications . . . . . . . . . 47

$3 \mathrm{~Pb}-\mathrm{Bi}-\mathrm{Sn}$-Cd-In physical properties $\ldots \ldots \ldots \ldots \ldots \ldots$ 


\section{List of Figures}

1 LM fast film limiter design $\ldots \ldots \ldots \ldots \ldots \ldots \ldots$

2 LM fast film divertor design $\ldots \ldots \ldots \ldots \ldots \ldots$

3 Coplanar divertor with bent substrate . . . . . . . . . 15

$4 \quad$ LM droplet limiter design $\ldots \ldots \ldots \ldots \ldots \ldots$

$5 \quad$ LM droplet divertor design . . . . . . . . . . . . 18

6 Geometry of LM film flow . . . . . . . . . . . . 21

7 Free surface height of $\mathrm{Ga}$ and $\mathrm{Li}$ films under the influence of a normal particle flux . . . . . . . . . . . . . . 24

8 Velocity profile for induced field model $\ldots \ldots \ldots \ldots \ldots$

9 Film geometry with incident particle flux . . . . . . . . 39

10 UCLA-MEGA loop diagram $\ldots \ldots \ldots \ldots \ldots$

11 Magnetic coils cutaway view . . . . . . . . . . . 48

12 4-coils set magnetic field magnitude per 1000 amps (6 in. spacing) 49

13 4-coil set lines of force $(6$ in. spacing $) \ldots \ldots \ldots \ldots$ 


\title{
ABSTRACT OF THE PROSPECTUS \\ MHD Modelling of Liquid Metal Films for Fusion Divertor Surface Protection
}

by

\author{
Neil B. Morley \\ University of California, Los Angeles \\ Professor Mohamed A. Abdou, Chair
}

In order to counter adverse effects resulting from the impingement of high energy plasmas on solid material surfaces, especially as this relates to fusion reactor high heat flux components, the idea of protecting the material surface with a thin film of liquid metal has been advanced. In principle, this film would protect the underlying substrate from physical sputtering and reduce thermal stresses in the structure. However, serious concerns related to establishing such a liquid metal flow and its performance in a fusion environment need to be addressed. In particular, the interaction of the conducting metal film with the complicated magnetic fields typical of a diverted reactor plasma may lead to retardation of the film resulting in channel flooding, velocity profiles not conducive to effective heat transfer, and possibly even detachment of the film from the substrate. In addition, the momentum carried by the plasma particles may deform the film shape to a significant extent, possibly disrupting the flow or leaving sections on the substrate inadequately protected. 
Proposed here are several mathematical and experimental models intended to address these specific questions. Mathematical models will be derived from the basic set of incompressible magnetohydrodynamic equations for the cases of fully developed and developing film flow. The fully developed flow model allows simplification of the governing equations to two dimensions, facilitating their solution. The data obtained from this formulation will yield the velocity, induced magnetic field, and height of the film as a function of space and flow parameters. From this data the effect of the plasma momentum on the shape of the surface will be seen, as will the velocity structure across the channel, a structure that is only assumed in previous modelling attempts.

The developing film model, based on simplifying assumptions for the height and velocity profiles determined from the previous model for the fully developed case, will account for spatial and temporal varying magnetic fields. In this way it will be possible to model more fusion relevant field distributions and establish their effect on the evolution of the film and its possible flooding or detachment as it flows along the substrate.

An experimental facility will be constructed for the investigation of wide film flows in strong coplanar magnetic field. Measurements of the film height and electric potential will be made that can then be used to validate the developing film model predictions. This experiment will involve films of larger aspect ratio than previously possible in narrow gap magnets, and will be extremely valuable to convincingly establish the physical possibility of creating the desired thin, wide films for fusion reactor, divertor/limiter surface protection. 


\section{Chapter 1}

\section{Introduction}

The use of a thin film of flowing liquid metal (LM) for the protection of the divertor surface was originally proposed in an effort to decrease the erosion damage and thermal stresses caused by high energy particle and heat fluxes incident on a solid material. As fusion designs evolve, it is clear that there is still no solid material capable of withstanding these loads to a satisfactory degree at the present estimated plasma edge temperatures of a fusion reactor. For this reason, development of a feasible divertor system is currently of pivotal importance in the area of fusion energy technology, and more attention is being given to alternative concepts, including the liquid metal thin film protection idea. This work involves the development of mathematical models that describe the effect of a magnetic field, plasma pressure, and gravity on a thin film liquid metal flow. In addition, an experimental LM facility and thin film test section will be built to help validate the models and explore new phenomena. These tasks are expounded on later this Introduction and explored in greater detail in Chapters 3 and 4. 


\subsection{Liquid metal divertor system issues}

The use of a flowing film of liquid metal in the capacity of protecting a solid substrate has several positive features that make the idea very attractive to further investigation. These features include:

- protect underlying surface from physical erosion and surface blistering,

- a continually replenished surface that replaces eroded material,

- a large heat removal capability that can eliminate the need for a separate coolant,

- and reduced heat penetration to the structure thus reducing thermal stresses and tile attachment problems.

Although film protection theoretically eliminates several of the existing problems in the development of solid divertor surfaces, the use of a liquid metal in contact with the fusion plasma scrape-off region adds problems uniquely its own. The feasibility of such a concept needs to be established and a capability to predict component performance must be developed.

Magnetohydrodynamic (MHD) forces, caused by the interaction of a moving conducting fluid with the confining magnetic field, can affect both the shape and velocity of the film as well as its free surface stability to perturbations. Plasma current disruptions may have catastrophic effects on the film due to the rapidly changing magnetic fields and the forces they induce in the film. Solving problems in this area requires using the normal fluid equations coupled with Maxwell's equations and can often be very difficult. 
The flux of particles incident on the divertor surface carries a certain amount of momentum (plasma wind) which will be deposited in the film. This momentum may be great enough to seriously influence the film shape and lead to the formation of waves or dryspots, leaving part of the solid substrate inadequately protected.

Evaporated and sputtered atoms may become ionized and diffuse back into the main plasma. These impurities, particularly for high atomic number metals, may critically contaminate the plasma because of high radiation losses. Estimation of the severity of this effect is difficult since the sputtering of liquid phase metals due to isotopes of hydrogen and helium, as well as their self sputtering and redeposition characteristics, may be different than their solid counterparts. This has not yet been deeply investigated. However, the problem of plasma contamination is common to both liquid and solid surface divertors

The solubility of tritium and helium in the liquid film can have negative results. A high tritium solubility can lead to a large tritium inventory in the liquid metal loop, especially if the metal is not treated for tritium removal. Also, for metals with low tritium solubility, the partial pressure of tritium may be so high as to push tritium through pipe walls and into other reactor areas, like the secondary cooling loop. With low solubility there is also the chance that implanted atoms of helium and tritium will form bubbles that burst in the chamber [1]. Again, similar problems exist for solid material surface divertors. The liquid metal film concept has the advantage of allowing the possibility of tritium removal from the circulating LM.

These issues must be addressed in order to determine the overall feasibility of the liquid metal protected divertor system. 


\subsection{Goal of the research}

It is the goal of this proposed research to describe the effect of the major MHDrelated influences on the film behavior. Models, both mathematical and experimental, will be developed to determine to shape of the surface and the velocity of a flowing film exposed to fusion reactor-like conditions. With this information it will be possible to draw conclusions on the system feasibility and attractiveness from the standpoint of MHD restrictions.

Because of the difficulty involved in solving the general three dimensional set of fluid equations coupled to Maxwell's equations, it is necessary to reduce the complexity of the system by making certain simplifying assumptions and exploring special cases. Two mathematical models of the liquid metal film are proposed in an effort to demonstrate the effect of specific mechanisms of the MHD and plasma coupling; in particular the effect of sidewalls in large aspect ratio ducts, the deposition of particle momentum on the surface of the film, and spatial and temporal varying magnetic fields. Exploration of these phenomena has only been done to a limited extent (see Chapter 2), and it is necessary to describe them in more detail if their influence on the film is to be accurately predicted. These models and their objectives are outlined below.

\section{Model 1 - Fully developed Film with Sidewalls}

- Determine the equilibrium height for a given set of flow parameters

- Determine the effect of a large aspect ratio $\left(\beta^{-1}\right)$ on the form of the flow

- Determine the relative importance of viscosity and electromagnetic forces on the flow 
- Show the effect of the momentum deposited on the surface by the particle flux

\section{Model 2 - Developing Film}

- Demonstrate the developing distance for different flow parameters

- Again show the effect of the momentum deposited on the surface by the particle flux

- Show the effect on the film of space varying magnetic fields of different orientation

- Show the effect of time varying magnetic fields, particularly for disruption simulation

- Find flow parameters that predict a usable film for a liquid metal protection system

These models are presented in the chronological order of their development. This distinction is made due to the fact that information revealed by the first model may be useful in the development of the second.

In addition to these mathematical models, an experimental LM facility and thin film test section allowing the simulation of a liquid metal protection system will be constructed and used to benchmark some important aspects of the mathematical results. This test section will have a larger aspect ratio than previous experiments performed in the Soviet Union in order to observe a more fusion relevant film. Measurements of the film height and electric potential will be made and used to assess the applicability of existing models. Also, other phenomena not 
included in the mathematical models will be observed in the experiment. These include engineering questions as to the performance of the film former and collector, as well as surface wetting. With the experimental setup in place, it will be possible to perform more detailed experiments and measurements as the situation warrants. 


\section{Chapter 2}

\section{Review}

In tokamak fusion reactors, it is desired to confine a burning deuterium/tritium $(\mathrm{D} / \mathrm{T})$ plasma within a toroidally shaped vacuum vessel with the help of a strong magnetic field. The objective of this confinement is to then use the energy produced by fusion reactions in the plasma to drive a conventional electric power station. However as the plasma burns, helium ( $\mathrm{He}$ ) ash builds up and must be removed. By scraping off the outer layer of the plasma, either with a limiter or a divertor arrangement, $\mathrm{He}$ and other impurities, as well as $\mathrm{D} / \mathrm{T}$, are continuously removed. But with both limiters and divertors, this high flux of energetic plasma particles collides with a surface intended to slow down and neutralize the energetic ions. These plasma contact surfaces are thus subject to high heat and particle fluxes which can have detrimental effects on conventional materials. As indicated in the Introduction, these contact surfaces can be eroded at an unacceptable rate [2] as well as suffer from high thermal stresses, material degradation, and blistering or cracking. As said before, it is in an effort to negate these effects that liquid metal contact devices have evolved. 


\subsection{Introduction to LM contact devises}

The idea of using a liquid metal in contact with a fusion plasma is not a new one. Over the years there have been many designs and much work devoted to addressing the questions and problems encountered in each design. The selection of one scheme is difficult to make until a more complete picture of the effects of the entire LM contact system on the other reactor systems is constructed. In order to gain a better understanding of the choices available, it is worthwhile to review past work on these LM systems.

\subsubsection{Choice of liquid metal}

A lengthy list of desirable characteristics for a liquid metal in contact with a fusion plasma has been compiled from a variety of sources. A general discussion of the comparison between metals is available in [3], [4], and [5]. A discussion of each of these criterion is provided to help understand its relative importance.

\section{Desired LM Characteristics}

- low melting and high boiling points

- low vapor pressure and sputtering erosion characteristics

- low atomic number $(Z)$

- compatibility of LM with structural materials

- compatibility of LM with other reactor materials

- high heat removal capability 
- low cost

- low activation by neutrons

- favorable hydrogen and helium trapping/releasing characteristics

There are certainly practical advantages to having a low melting point for the choice metal. The complication of pipe heating to maintain a liquid phase can be avoided, as well as concerns of pipe and pump damage due to volume increases of the metal upon solidification. A high boiling point provides an initial upper bound on the usable temperature window. As will be seen later, a large window is important in determining the heat removal capability of a LM candidate.

In order to minimize the number of impurity atoms that leave the metal surface and diffuse into the plasma, it is important to limit the allowable evaporation and sputtering fluxes. Thus, low vapor pressure and sputtering characteristics are desirable. It has been suggested that the upper bound on the temperature window is determined by the temperature at which evaporation becomes unacceptable. Estimates of evaporative and sputtering fluxes are given in [5] and [6].

Once an atom has entered the plasma, it is desirable to minimize its negative effect. Since the radiative losses increase with $\mathrm{Z}$, a low atomic number for the impurity atoms is important, especially in the pre-ignition situation. However, often high $\mathrm{Z}$ materials have a higher threshold for physical sputtering and hence less material erodes and enters the plasma. The selection of material must be made to optimize the combined effect on the plasma.

Another possible limit on the maximum film temperature is the restrictions imposed by the compatibility of the LM with its structural material. At elevated 
temperatures, unacceptable corrosion of the structure can occur and lead to contamination of the LM and mechanical failures of the circulation system. Estimates for corrosion limitations of various LM with various structural materials is provided in [6] and to a lesser extent in [7]. Also important in some designs is the wetting characteristics of the LM. In order to insure a proper flow the LM may need to wet its substrate [7].

In order to avoid potential safety problems it is important to have the LM compatible with other reactor materials, even those which might not be in intimate contact with the LM under normal conditions. The most common examples are air and water. Metals that are extremely reactive with these compounds would be poor choices from a safety point of view unless much care is taken in the system design.

The ability of a specific LM to carry out incident energy in the form of heat is especially important for self-cooled system designs. The suggested criterion to measure this ability is the value of the factor $\Delta T \sqrt{\rho c k}$, where $\Delta T$ is the usable temperature window, $\rho$ is the density, $\mathrm{c}$ is the specific heat, and $\mathrm{k}$ is the thermal conductivity of the LM [8]. A comparison for several metals is given in [9]. Also important in this area is the ability of the metal to withstand plasma disruptions. This ability is characterized in [5] as the minimum thickness of a film necessary to protect the underlying surface. However, this characterization is not applicable to droplet designs (for description of droplet design options see Section 2.1.2).

Low cost certainly requires no explanation. Obviously, rare and/or expensive materials will add to the overall reactor cost as well as present problems with supply. 
Again from a safety standpoint it is desired for the candidate metal to have little neutron-induced activation. Some activation is inevitable, therefore, the half-life and type of decay must also be investigated. Information on activation of different metals in a fusion environment is included in [3].

It would be very helpful indeed to find a liquid metal with high helium and low hydrogen $(\mathrm{H})$ absorption characteristics to facilitate the pumping of He and the recycling of $\mathrm{D} / \mathrm{T}$. Although the solubility of He is low in most metals, the solubility of $\mathrm{H}$ varies drastically in the different choices. This solubility and the LM's ability to form chemical compounds with $\mathrm{H}$ provides an estimate to how much $\mathrm{H}$ will be removed with the liquid. The tritium inventory in the LM volume is highly dependant on the individual LM properties. It has been shown that both high and low recycling divertors are possible depending on the system design and the metal chosen $[1,3,10]$.

Of the available metals and alloys, the two choices most commonly considered are lithium ( $\mathrm{Li}$ ) and gallium (Ga). Specific information on these two candidates are provided by the citations above. In general, $\mathrm{Li}$ is characterized by an affinity for hydrogen, high vapor pressure, high melting point, and poor heat removal capacity as compared to Ga. However, Ga has more significant corrosion problems, higher $\mathrm{Z}$, worse activation characteristics, and is much more expensive than $\mathrm{Li}$. Selection of a given metal must depend not only on the criteria above but also on the specific design and desired plasma edge conditions.

\subsubsection{Designs of LM plasma contact systems}

Many different LM Plasma Contact Systems designs have been proposed in an effort to minimize the problems, as well as to take maximum advantage of the 
strengths, associated with the use of a LM as a contact surface. In general, these designs break down into three catagories: films, droplets, and jets.

\section{LM film designs}

Film designs operate by covering a backing plate with a film of liquid metal. This film then protects the surface below from the effects of the high energy particle flux coming from the main plasma. Film designs can further be broken down into fast and slow films categories [11]. The former must remove all incident energy as well, while the later serves only as protection with a separate coolant responsible for the heat removal.

Fast film designs were included in the UWMAK-I [12] and as an advance concept in the INTOR [13] design studies. They have the added advantage of eliminating the need for separate coolant and thus reducing thermal stresses in the structure. Disadvantages come from the fact that a greater velocity or a thicker film is generally required to remove the heat. Greater velocities mean more MHD and corrosive effects while thicker films lead to larger loop volume and tritium inventory. However, the minimum thickness estimated for disruption protection by Hassanien and Smith [5] is of the order of 1-2mm, depending on the LM used. It is possible to use a $2 \mathrm{~mm}$ film as a fast film device [14] so that no thickness changes are required. More complete fast film designs are proposed by the Soviet Union for use in the International Thermonuclear Experimental Reactor (ITER) [4]. Examples of fast film limiter and divertor designs are shown in Figure 1 and 2.

Slow films have also been investigated [5]. Because of reduced velocity these devices do not suffer as significantly from negative MHD effects. The separate 


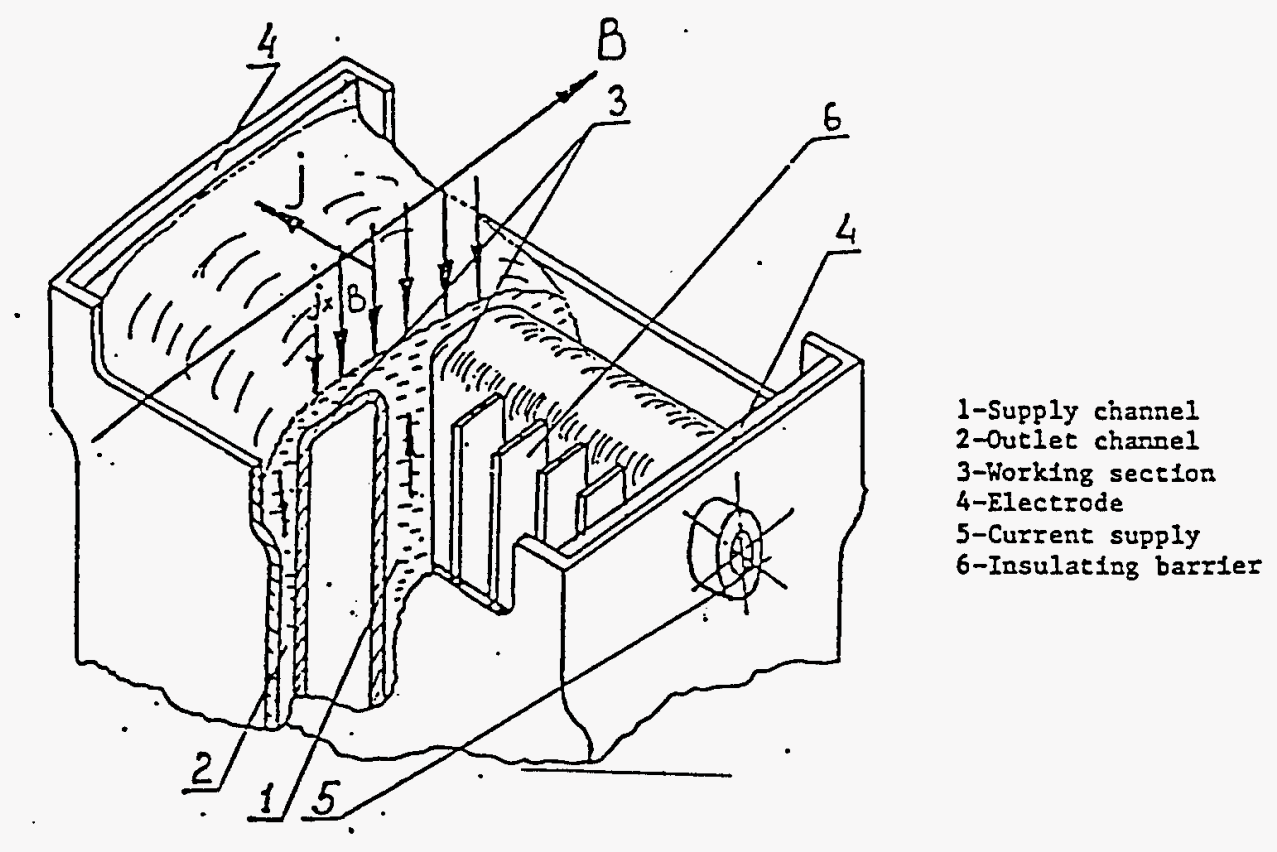

Figure 1. LM fast film limiter design

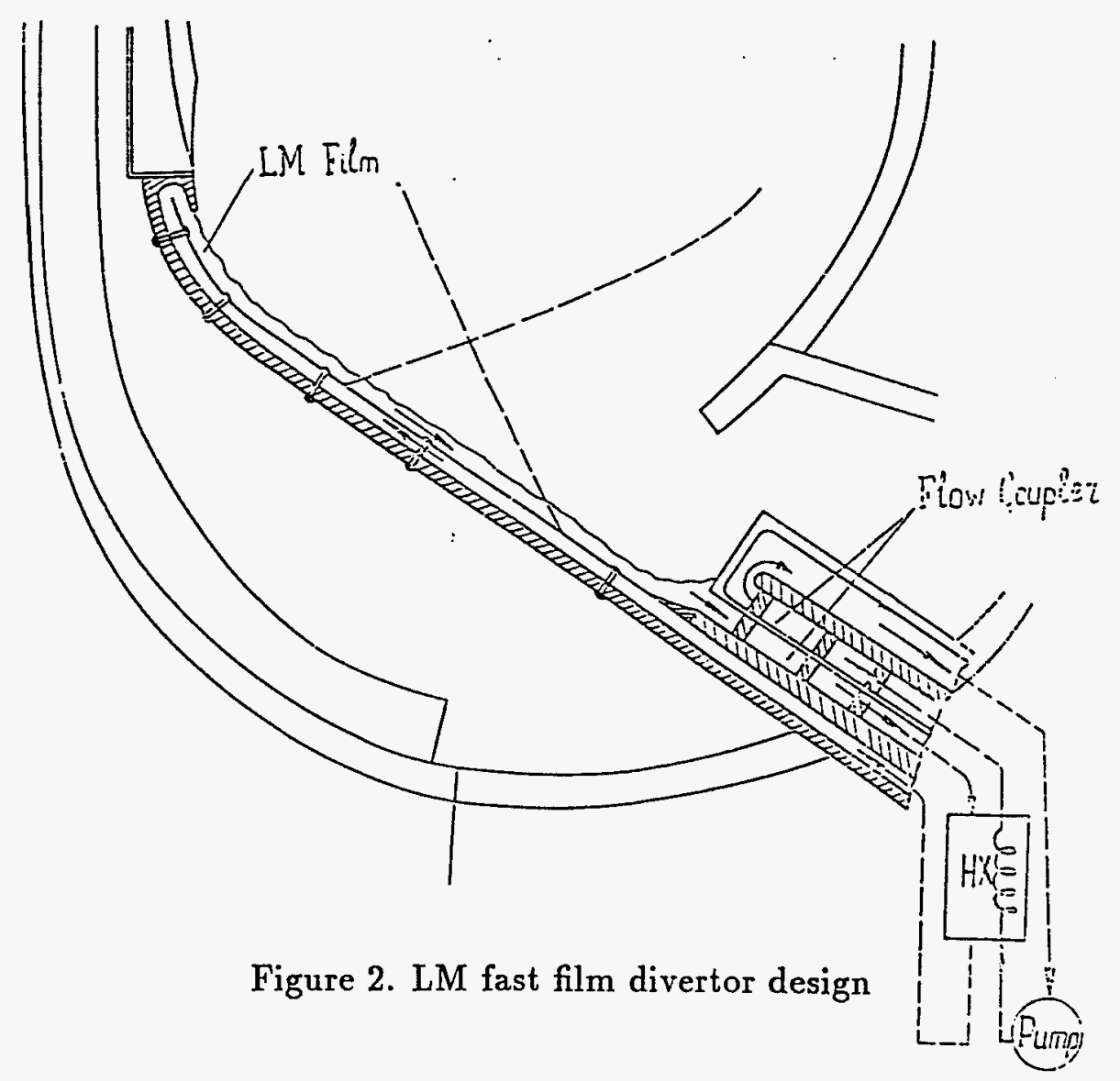


coolant must still be run at a high enough rate to keep the maximum film temperature below whatever limit is imposed (see Section 2.1.1). From a safety point of view there is a trade-off here between the likelihood of a failure of LM flow in a fast film or of the coolant in a slow film. In the slow film case, however, the substrate is still protected for a time after a coolant failure even though the LM film temperature continues to rise.

For quasi-coplanar field divertor designs typical of Figure 2, a question about the presence of sidewalls becomes important. If the sidewalls protrude above the level of the liquid, they will be exposed to the high particle flux and thus eroded very quickly. This eroded material will contribute to the plasma impurities. In order to avoid this several designs have been suggested.

1. Removal of sidewalls entirely; the flow will be ither a continuous ring or broken up with catch trays in the gaps

2. Slight flooding of the channel; liquid film will cover over the top of the sidewalls

3. Bending the substrate; the walls will lie in the shadow of the particle flux (see Figure 3)

Comparisons of these ideas, and determination of the most likely candidate, has yet to be done.

Both slow and fast film designs involve LM flow over a substrate in a magnetic field. Experimental investigations of MHD film flow have been performed in order to confirm the possibility of creating the needed films in the presence of a magnetic field. A module demonstrating flows similar to Figure 1 was described in [15] and [16]. These tests showed the formation of an M-shaped surface structure at the 


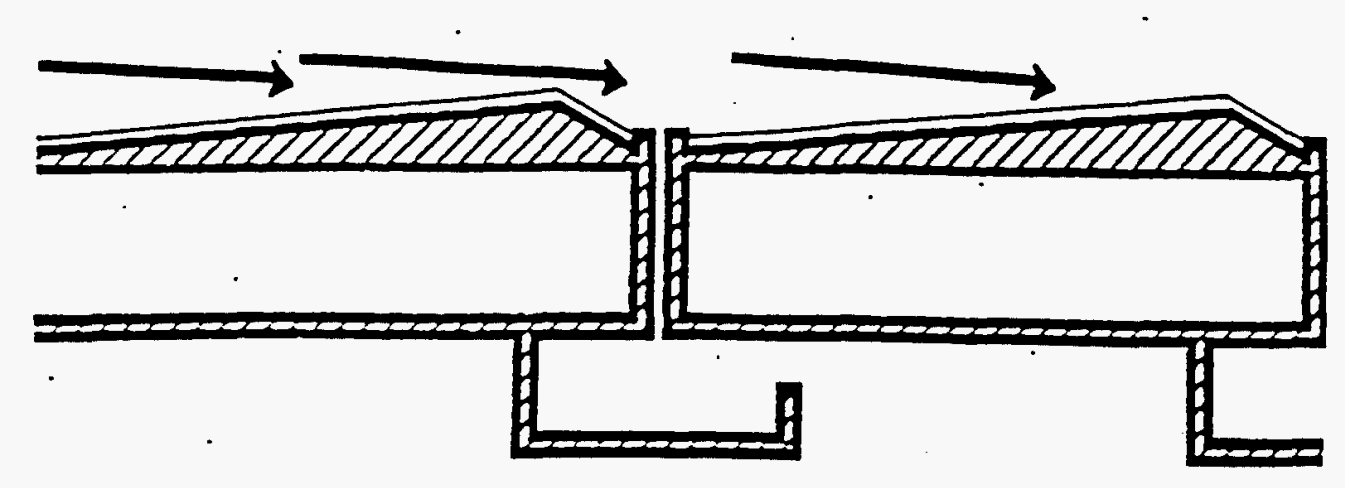

Figure 3. Coplanar divertor with bent substrate

inlet and the occurrence of channel overfilling at the outlet, both due to MHD effects. These phenomena were characterized in terms of the flow parameters and ways of avoiding them are described. Flows characterizing the geometry of a divertor, like the one in Figure 2, were investigated in [7], [17], and [4]. It was determined that for complete filling of the backing plate as well as a minimum of MHD interaction, it is desirable to use a wetted, weakly conducting backing plate with non-wetted, poorly conducting sidewalls. Under these conditions thin film flows were demonstrated with velocities up to a $5 \mathrm{~m} / \mathrm{s}$. Also demonstrated was the suppression of surface waves and disturbances, and the ability to flow at angles over $90^{\circ}$ (flow on the ceiling) [7], confirming in principle the possibility of an upper divertor [4].

\section{LM droplet designs}

LM droplet contact systems operate by filling the plasma contact area with an impenetrable collection of LM droplets, absorbing the particles and energy. Droplet 
designs have the advantage of little droplet interaction with the magnetic field [8] and are inherently low recycling and self-cooling $[10,6]$. Because of the reduced interaction with the field, the droplet designs are less sensitive to off-normal reactor conditions, including disruptions. Disadvantages include an increased complexity of design and a larger volume. These systems are generally subdivided into three parts: a jet droplet former, interaction region, and a droplet collector.

The formation of droplets can be accomplished outside of the magnetic field and injected into the vacuum chamber $[10,18]$, or inside the chamber itself $[6$, 3]. The former case requires space in the tritium breeding blanket for injection vacuum ducts, reducing availible breeding space; and involves the droplets passing through a region of non-uniform magnetic fields, which can cause elongation of the droplets and reduced coverage of the contact area $[19,20]$. In the later case, a compact droplet former (DF) that can operate in a high magnetic field must be developed. Such a DF design, using the alternating electromagnetic forces to stimulate the break up of LM jets, is described in [21]. High velocity jet formation in the magnetic field may also involve large MHD pressure losses in the feed loop and DF.

In order to completely cover the interaction region, the droplet cloud must be several layers thick. Velocities on the order of $10-20 \mathrm{~m} / \mathrm{s}$ [3] will be required to remove the incident energy without the droplet temperature exceeding its maximum (see Section 2.1.1). It has been estimated that the effect of the plasma wind on the droplets will cause a displacement of approximately $4 \mathrm{~cm}$ in the toroidal direction and $5 \mathrm{~mm}$ in the radial direction [6]. Provided the collection system is designed for this shift, it should pose no problem to the feasibility of the droplet concept. Due to the engulfing action of the droplet system, a significant amount 


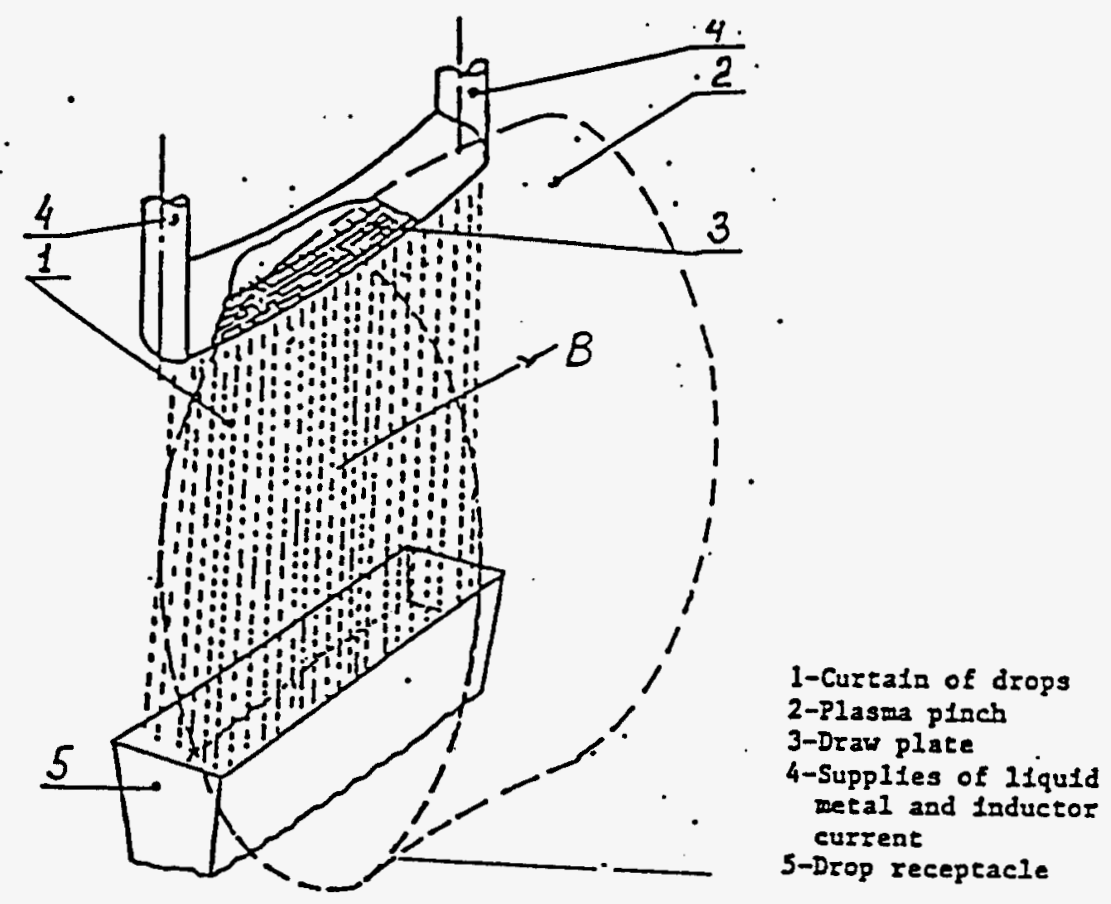

Figure 4. LM droplet limiter design

of particle pumping is expected $[22,10]$. It is possible, however, that some of this gas might be liberated when the droplets collide with the collector surface.

The droplet collector must gather the droplets as they leave the interaction region without allowing significant splashing of the metal, and drain the liquid to the outlet, where it may be cooled, degassed, and recycled to the inlet. These collectors typically are just troughs partially filled with LM. Figures 4 and 5 show droplet limiter and divertor designs.

Several experiments have been performed to verify droplet design feasibility. Studies of droplet impact on a pool of liquid metal show that the magnetic field completely suppresses splashing $[9,4]$, thus validating the simple trough concept. Experiments with the droplet former have demonstrated its successful operation [21]. Also performed were plasma physics experiments in the Soviet T-3 tokamak where a liquid metal droplet curtain was used as a limiter similar in configuration to Figure 4. The behavior of the LM limiter was compared with a graphite 


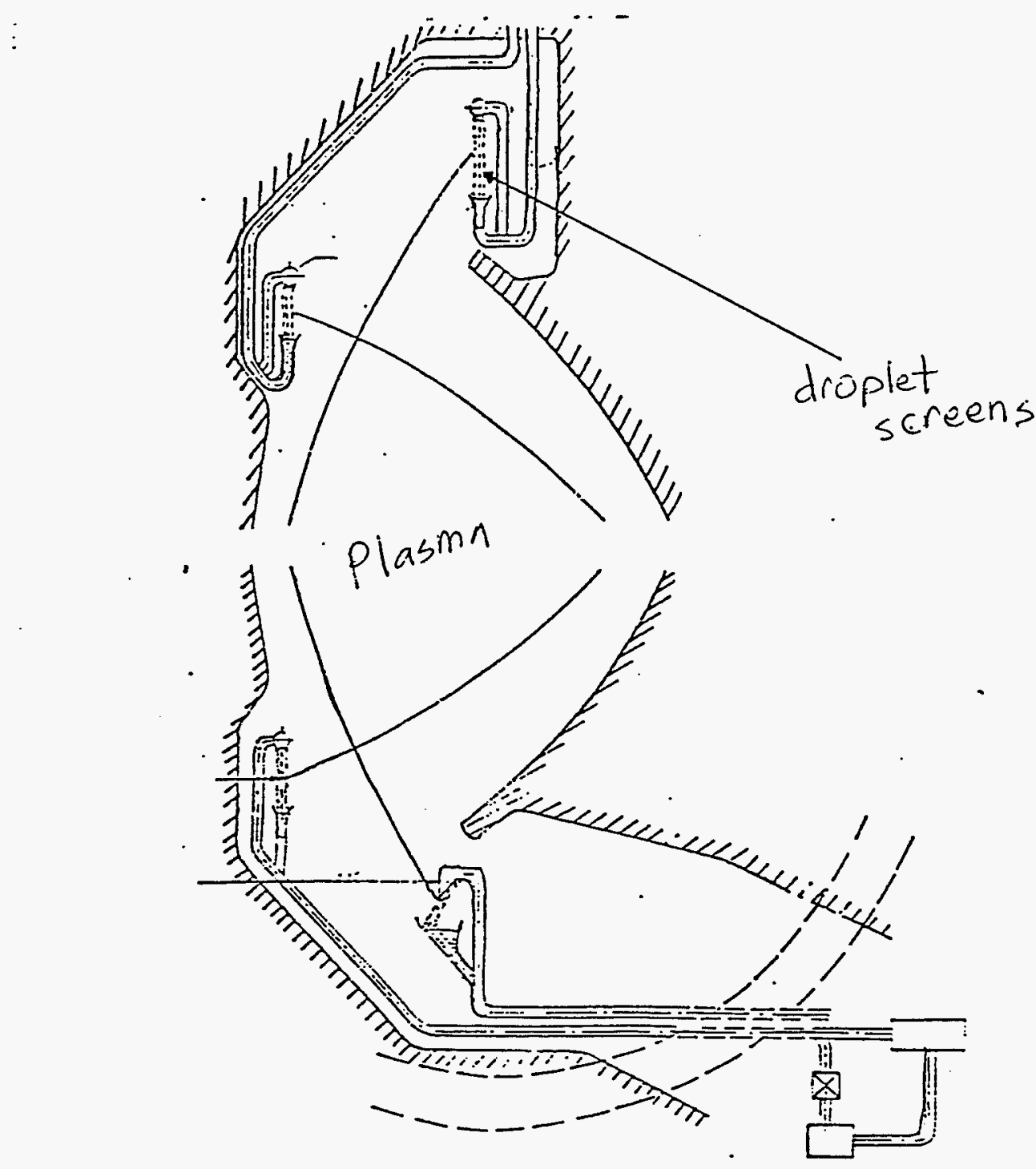

Figure 5. LM droplet divertor design

18 
control limiter of similar geometry. Tests showed that a discharge was possible with plasma parameters similar for each limiter. However, the LM limiter in the initial stages of discharge showed high radiation losses from the plasma attributed to increased impurities, and significant spraying of the LM around the vacuum chamber [23]. It is claimed that the impurities were largely the result of improperly treated LM for the removal of dissolved gasses. The spraying of the LM is attributed to physical swaying of the DF due to the pulsed nature of the main magnetic field coupling to the inductor of the DF. With their trajectories diverted by this swaying, some droplets failed to exit through the small exit slot at the bottom of the vacuum chamber [22]. Experiments to verify these conclusions are being built.

\section{LM jet designs}

There is not much to say about jet designs that has not already been said about films and droplets. Jets, by nature, are a middle ground between the other two options. They do not have as significant MHD interaction characteristics as films, but more so than droplets. On the other hand, they are more complicated to produce than films, but not so much so as droplet designs. In general, though, there is not much literature available on this type of system and they are currently not considered as options as often as the other two.

\subsection{Mathematical models of MHD film flow}

Since the work to be proposed in this prospectus lies primarily in the area of mathematical modelling of LM thin film flows in a magnetic field, it is worthwhile 
to look in greater detail at previous modelling efforts. In order to fully understand the problems discussed it is necessary to be familiar with magnetohydrodynamic effects, especially as they apply to incompressible fluids. A review of these topics is available in [24] and [25]. Most models for MHD film flow are mathematical equations that predict the height of the film as it evolves down the channel. Some more sophisticated models attempt to solve for the velocity profiles as well.

Alpher et al. [26], in addition to performing experiments concerning liquid mercury flowing in a transverse magnetic field, performed some simple modelling as well. By assuming no changes in the film velocity or height in the longitudinal and coplanar directions (see Figure 6), Alpher calculated a Hartmann velocity profile over the depth of the film. In addition, the induced fields and currents are calculated with the amount of current entering the wall based on the wall conductance ratio $\Phi=\frac{\sigma_{w} t_{w}}{\sigma_{f}} h_{f}$, where $t_{w}$ and $h_{f}$ are respectively the thickness of the bottom wall and height of the fluid and $\sigma$ is the electrical conductivity. However, the classification of the pressure gradient as a driving force for the flow and the estimates of its value, are erroneous conclusions based on the assumed restriction that $h_{f}$ does not change along the longitudinal direction. In reality the a nonchanging film height (fully developed flow) can only be achieved if an external body force, like gravity or an applied electic current, is present to offset the viscous drag and retarding MHD forces.

As interest in LM film flows for fusion increased, there became a need to more accurately predict the surface contour and velocity profile. UWMAK-I [12] assumed a completely coplanar (see Figure 6 ) magnetic field, one component ( $\hat{x}$ ) of velocity, and infinite duct length in the $\hat{z}$ direction. With these assumptions it was concluded that for the case of a fully developed film, there would be no retarding 


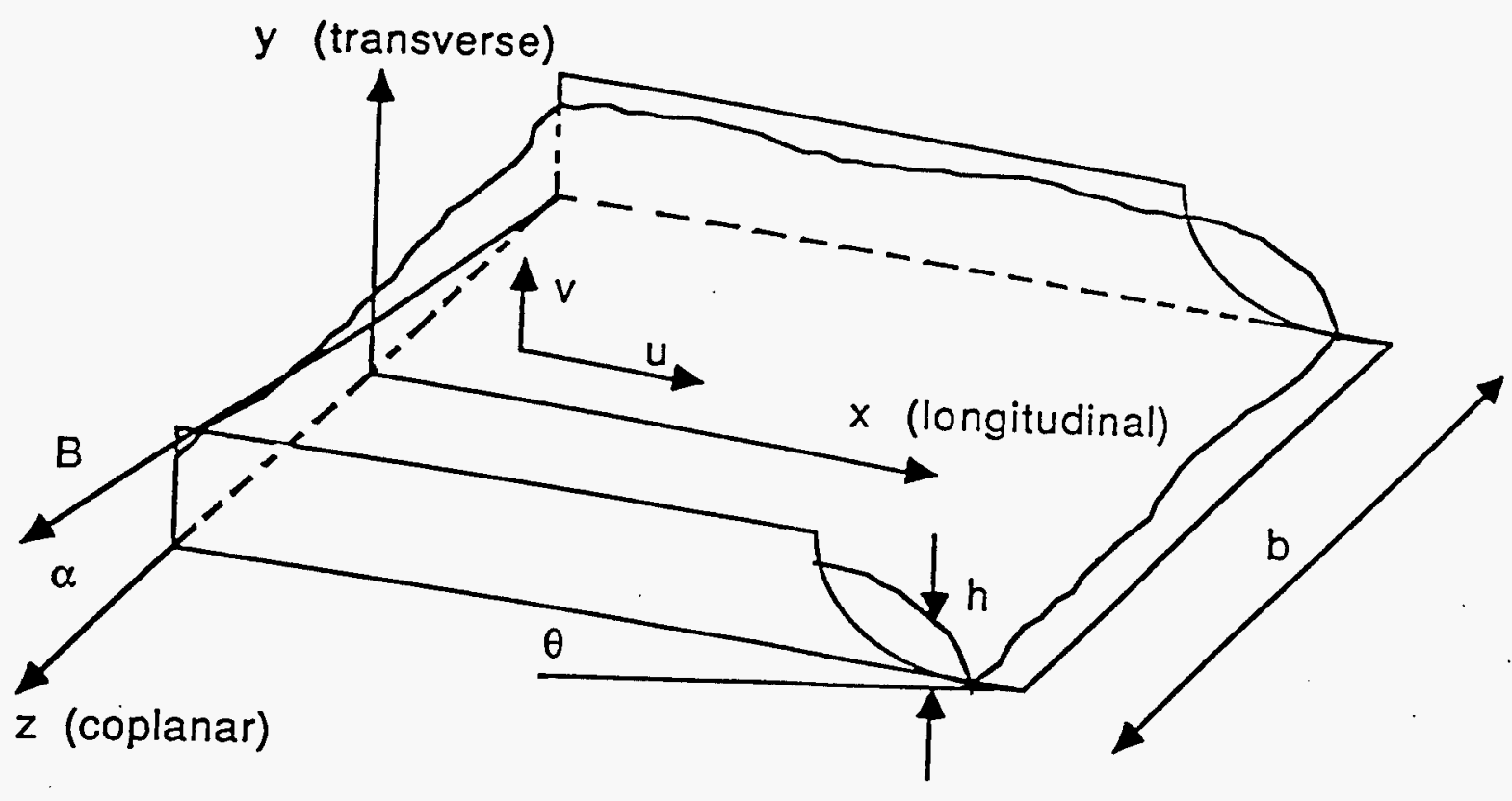

Figure 6. Geometry of LM film flow

force since $\vec{V} \times \vec{B}$ is perpendicular to the plate and constant over its area. This means there will be no return path for the induced current and therefore no current will flow. For the case of developing flow this constancy is no longer true but the integral of $\vec{V} \times \vec{B}$ over the film height is still constant, resulting from the constant flowrate condition (see page 35 ). Therefore, to a first approximation the bulk fluid is not affected by the magnetic field. Due to complicated field configurations in the divertor area, however, it is quite likely that there will be field components in the transverse direction (quasi-coplanar), leading to a significant field/film interaction predicted by Alpher and shown more specifically by Wells [18]. The effect of the presence of sidewalls is discussed later.

In attempts to describe the quasi-longitudinal field geometry indicative of limiter designs like the one in Figure 1, several models have been developed. Murav'ev and Yakovlev [27] obtained an expression for film height based on a conservation of energy approach. This again was for an infinitely wide duct. Later, Aitov and Kirillina [28], Murav'ev [29], and Morley [14] developed a one-dimensional 
estimate of film height using the set of MHD equations (see page 27). These onedimensional models rely on assumptions made about the distribution of velocity in the $\hat{z}$ and $\hat{y}$ direction and on the presence of sidewalls.

Since a divertor contact unit is planned for ITER, coplanar divertors like the one in Figure 2 are the current favorite fast film designs and thus receive greater attention here than the limiter models described above. Aitov and Ivanov [30] devised a one-dimensional model for film height from the MHD equations assuming a Hartmann profile of velocity and no fluid motion in the $\hat{z}$ direction, and a parabolic velocity profile in the $\hat{\mathrm{y}}$ direction. The shallow water approximation is used to eliminate terms of order $\left(\frac{h_{f}}{b / 2}\right)^{2}$ as small since the film is very thin compared to the channel width. The solution method employed was to average the governing MHD equations over the width, solve the $\hat{y}$ component of the equation of motion for the pressure, and substitute it into the $\hat{x}$ component equation. The velocity is then eliminated by expressing it in terms of a constant flowrate and integrating the resulting equation over the thickness of the film. Thus they were left with an equation for $\frac{d h}{d x}$ in terms of $x$ only. For some situations with small $\frac{d h}{d x}$, this equation matched up fairly closely with experimental data [31]. Similar models were constructed by Murav'ev [29] and Morley [14] with different assumptions, inclusive of other factors, and with greater discussion of the phenomena predicted by the models. For instance, Murav'ev estimated the effect of a plasma wind for a channel with no sidewalls and discussed the possibility of the magnetic forces causing the film to detach from the substrate and splash around the vacuum chamber. Morley discussed the conditions for the film to tend towards a stable equilibrium thickness and examined ITER type applications of the model.

Recently Liao [32] performed a similar treatment including a development of 
the effect of the normal particle flux on the free surface. He showed that the effect of the particles pushing down on surface is always greater than the tendancy of the positively charged plasma to pull up on the negatively charged free surface. However, he concludes in the analysis that the total effect exerts a negligible influence on the film height and free surface stability.

Still more detail was necessary to determine the importance of other factors affecting a coplanar film flow. Evtushenko and Smoletzev [33] devised twodimensional models for the cases of a strictly coplanar film with sidewalls, and a three component field with no sidewalls. For the first case, a Hartmann profile is assumed in the $\hat{z}$ direction and the equations are averaged over it. Following a proceedure similar to Aitov's described above, a system of equations is found for $u$ and $v$ as a function of $x$ and $y$. The complexity on this system required its solution by numerical methods. The duct in this case is non-conducting. Regimes of decay and lift (of the film height) were classified as a function of the flow parameters and the tendancy of the developing length to decrease with increasing magnetic field was noted. Also characterized was the normal momentum flux on the surface of the film (the coplanar flux could not be included in this 2-D model). This effect was not significant for gallium but was very serious for lithium because of its lighter mass. Figure 7 shows the evolution of the film under the influence of a momentum flux that is stronger in the middle region. This result conflicts with Liao's assumption that this momentum flux was negligible.

The second case, with an infinite film in the $\hat{z}$ direction, allowed the assumption of $\frac{\partial}{\partial z}=0$. Again a two dimensional system in terms of $\mathrm{x}$ and $\mathrm{y}$ was developed. This model demonstrated the formation of a Hartmann velocity profile in the $\hat{y}$ direction when a transverse field is present, as was predicted by Alpher and Wells 


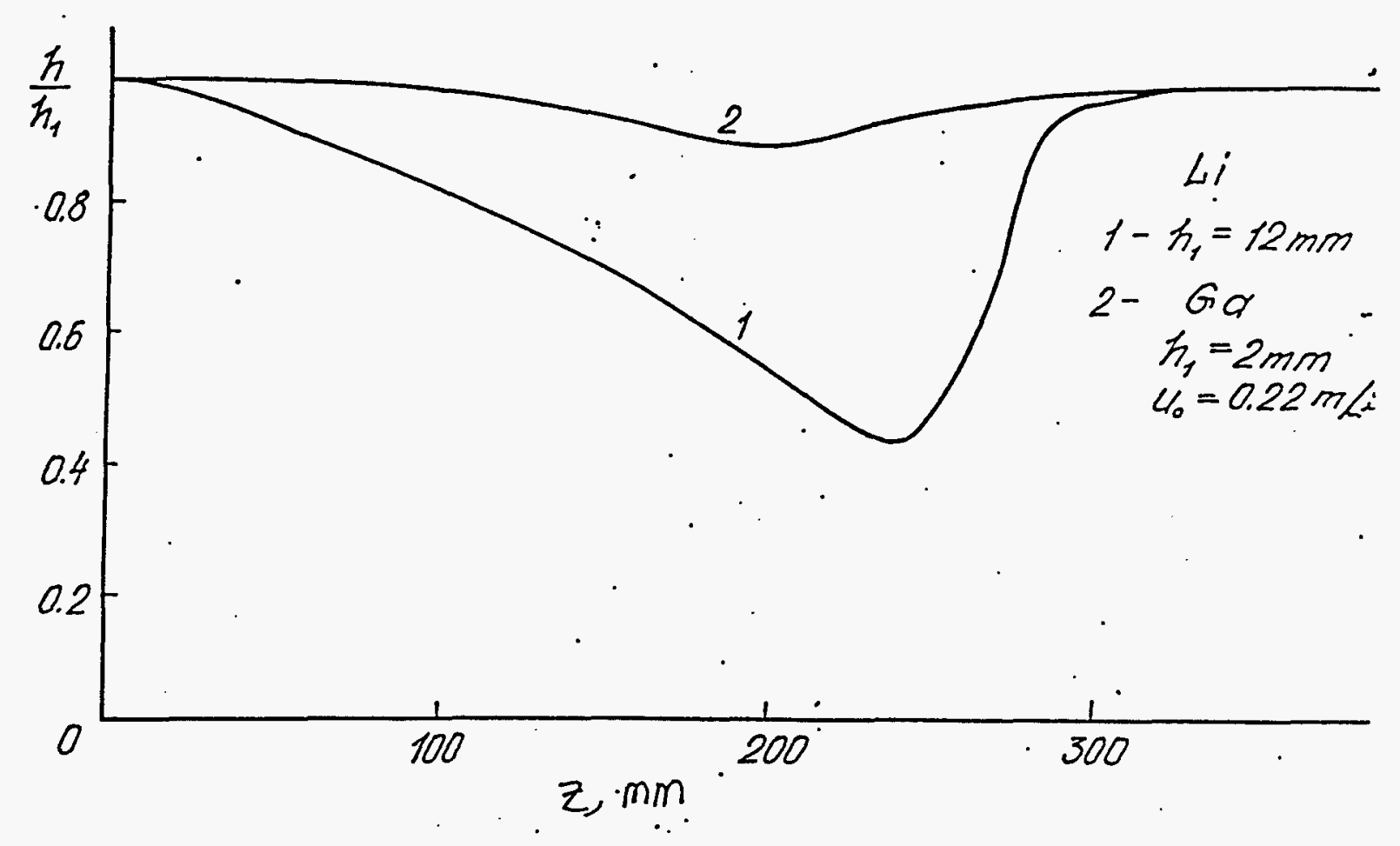

Figure 7. Free surface height of $\mathrm{Ga}$ and Li films under the influence of a normal particle flux

earlier (see page 20). Also allowed for in this model was the effect of a changing magnetic field. Control of the free surface by changing the field was demonstrated.

Further enhancements of these models, by including wall conductivity, is underway [31]. A reformulation of the problem in terms of the induced magnetic field, with the equations still averaged over a Hartmann profile in $\hat{z}$, is currently yielding results. This method predicts the formation of a velocity profile (in $\hat{\mathbf{y}}$ ) with a hollow area in the middle (see Figure 8). This is not seen in velocity/potential formulations but shows up with the velocity/induced field approach. Also under development is a fully developed, two-dimensional model for the velocity in terms of $y$ and $z$ coordinates [34]. These equations, instead of being solved numerically, are fitted to an assumed profile in the $\hat{z}$ direction. This profile is a combination of Hartmann and parabolic with coefficients selected to minimize errors. This 


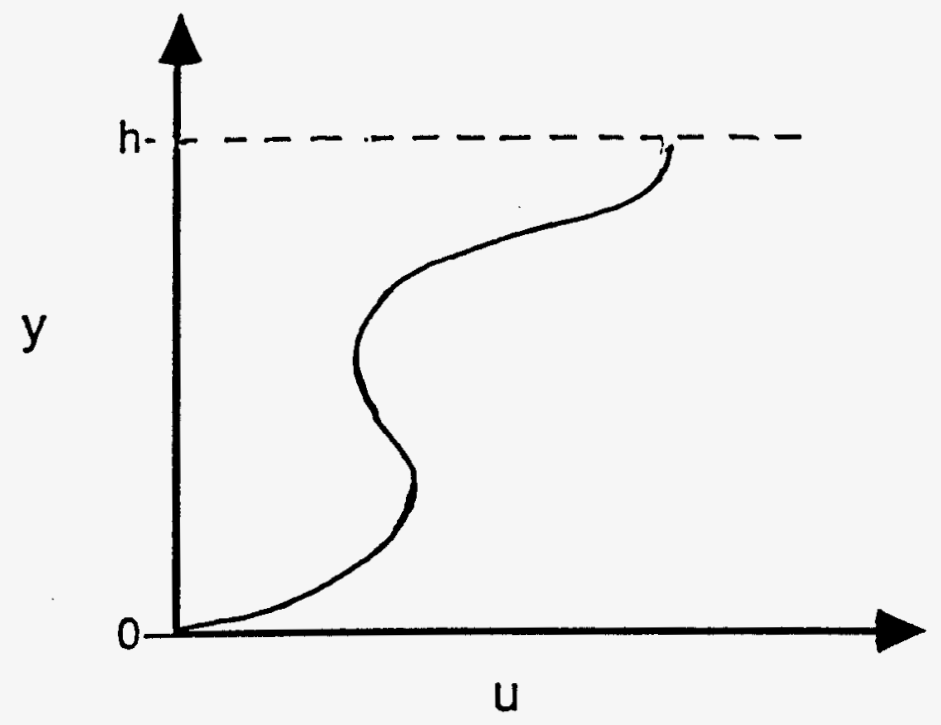

Figure 8. Velocity profile for induced field model

method again uses induced fields and describes a velocity profile with a hollow.

This previous work has established the general trends of the fast film devises, but major uncertainties still remains due to details of engineering and operating conditions of a real reactor. The models proposed in this work (see Chapter 3) will extend the present level of detail to better describe more fusion relevant flow situations. Also, conflicting conclusions about the effect of the particle flux and conducting sidewalls will be resolved. 


\section{Chapter 3}

\section{Proposed mathematical models}

In order to predict the behavior of liquid metal films in a fusion environment, it is necessary to develop models taking into account physical factors that may be of importance to the evolution of the flow. In this way it is possible to predict the relative effects of these factors and use appropriate ones in the development of more sophisticated models. This process will lead to a fairly detailed description of the film that can be used to establish the possibility of the use of LM films as plasma contact surfaces, and the flow parameters that result in such a flow. In this way also, a greater understanding of the physics of the problem is developed that will be advantageous in the actual design of verification experiments and the full-scale LM contact systems themselves. Given in the previous chapter was a summary of the work in this area. In this chapter is presented the extension of this work to the next step for quasi-coplanar divertor geometries, $\vec{B}=\left(0, B_{y}, B_{z}\right)$.

The models proposed here are derived from the basic set of magnetohydrodynamic equations. These equations include the Navier-Stokes equation, the mass continuity equation, the current conservation equation, Ohm's Law, and Maxwell's 
equations .

$$
\begin{aligned}
\frac{\partial \vec{V}}{\partial t}+(\vec{V} \cdot \nabla) \vec{V} & =-\frac{1}{\rho} \nabla p+\nu \nabla^{2} \vec{V}+\vec{g}+\frac{\vec{J} \times \vec{B}}{\rho} \\
\nabla \cdot \vec{V} & =0 \\
\nabla \cdot \vec{J} & =0 \\
\vec{J} & =\sigma(\vec{E}+\vec{V} \times \vec{B}) \\
\nabla \times \vec{B} & =\mu_{m} \vec{J} \\
\nabla \times \vec{E} & =-\frac{\partial \vec{B}}{\partial t} \\
\nabla \cdot \vec{B} & =0
\end{aligned}
$$

The definition of symbols is summarized in an appendix if reference is needed, but in general all quantities are symbolized with fairly standard notation. Here $\vec{V}$, $\vec{J}, \vec{E}$, and $\vec{B}$ are respectively the velocity, current density, electric, and magnetic fields. $\vec{g}$ and $p$ are the vector acceleration of gravity and the pressure; while $\sigma, \rho, \nu$, and $\mu_{m}$ are the electric conductivity, mass density, kinematic viscosity, and magnetic permeability of the material. Approximations implicit in the above system include incompressibility of the fluid and small displacement current [25, 35]. The alignment of the coordinate system is that shown in Figure 6 of the last chapter. Any deviation from this will be noted when appropriate.

\subsection{Remaining questions}

Of the many factors influencing the LM flow, several important ones are still left to a large extent unexplored, while others are involved in some disagreement. This work will address questions associated with:

1. large aspect ratio channels with conducting sidewalls and substrates, 
2. plasma particle flux on the free surface, and

3. space and time varying magnetic fields.

To some extent these have been investigated before, but a more complete treatment is warranted to firmly describe their effects. These factors represent the main MHD related issues that need to be concidered for relevent fusion environment modelling. With this done it will be possible to ascertain more precisely the possibility of establishing the desired flow in a real reactor situation, and the design parameters that lead to such a flow.

Conducting Sidewalls and Substrates - In some of the models introduced in the last chapter sidewalls effects were explicitly mentioned. This was because these models predicted that to a large extent the presence of sidewalls and their conductivity would greatly influence the shape of the free surface $[30,14]$, the velocity profile [33], and the free surface stability with respect to small perturbations $[36,32]$. Others have theorized that sidewalls, for channels where the coplanar length along the field is great compared to the thickness of the film, will not have significant influence on the bulk of the film [22]. This idea would lend support to models that do not include sidewall effects $[29,33]$. It is possible that the large sidewall effects are the result of averaging the governing equations over the width of the film with an assumed profile (Hartmann in this case) derived from duct flow situations with aspect ratio of order unity. For the specific examples [30] and [14], the electric field is assumed not to change from the bulk to the wall, i. e. not changing along the magnetic field. This is a common duct flow approximation [24] but is not applicable to large aspect ratio cases where, due to the absence of a top wall, the current must close through the film. Thus the effect of the currents in 
retarding the bulk may be over emphasized. Still these currents will not be zero and it becomes necessary to determine more precisely their influence on both the bulk film and flow near the sidewalls.

It should be noted that the presence of sidewalls in an actual divertor design is not necessarily a given. Because sidewalls that protrude above the level of the liquid film will be exposed to the particle flux, they will be eroded very quickly. Design ideas to counter this action are given in the previous chapter (see section 2.1.2). The flooded film and tilted substrate ideas are electrically similar to the simpler geometry proposed here.

Plasma Particle Flux - The second proposed factor in need of investigation is the effect of plasma momentum on the LM distribution and velocity. The momentum that is carried by the particle flux must be conserved and may be significant enough to considerably alter the form of the free surface. Some question as to the angle of incidence of this momentum makes it difficult to simply model its influence on the film. When a plasma is in contact with a structure (e. g. a probe, divertor or first wall), a potential difference forms between the two. The area of rapid change in potential close to the surface is known as the plasma sheath [37] and it acts to accelerate the ions (decelerating the electrons) normal to the free surface. However, in the pre-sheath area the charged particles tend to follow the magnetic field lines, which are nearly tangential to the plane of the film. This tangential momentum is conserved through the quasi-collisionless sheath. The final trajectory and magnitude of the momentum is an issue of some debate, and highly dependant on the plasma parameters.

The models that attempt to describe this effect do not fully treat the problem. Evtushenko and Smoletzev [33] can only account for the transverse momentum 
since their equations are averaged over the coplanar direction. Murav'ev [29] computes an estimate of the velocity of the film in the coplanar direction, but this is done without sidewalls, and assumes that the momentum is incident along the field lines and is deposited uniformly over the film thickness. Liao [32] concludes that the momentum is essentially fully normal and does not affect the film. Since this issue is thought of as one that could possibly exclude the use of LM films as a divertor surface, it seems necessary to more quantitatively examine this problem and realistically determine the effects that the particle momentum will have on the shape of the film.

Spatial and Temporal Varying Fields - Lastly mentioned above was the effect of varying magnetic fields. Accurate modelling of a fusion divertor environment requires that the space varying magnetic fields are accounted for as their influence is important to the evolution of the film. It is seen in $[14,33]$ that the fully developed equilibrium film height, i. e. the height to which the developing film tends, is estimated to be strongly dependant on the magnitude of the coplanar (toroidal) field. This field changes as $1 / R$, where $R$ is the major radius of the toroid; indicating a change in film tendacies as well.

Rapid changes in the poloidal magnetic field, like those during plasma disruptions, can induce significant forces in the film that may cause it to detach from the substrate and splash about the vacuum chamber. Recovery from such an event is also important. Effect of start-up and shut-down for pulsed systems must also be considered. Effort to include these effects must be made since the response of the film to these varying fields is one of the most significant MHD concerns with the use of the film option, as opposed to a droplet design (see Section 2.1.2). 


\subsection{Model-1: fully developed film}

A fully developed film means that there are no changes in the film status as it proceeds along the substrate, $\frac{\partial}{\partial x}=0$. Thus it is possible to develop a two-dimensional model for the film variables as a function of the $y$ and $z$ coordinates. It is convenient to break this model up into separate cases: flows with and flows without an impinging particle flux. The difference between the two in their development comes down to enforcing different boundary conditions on the velocity at the free surface. This difference can be determined by introducing the idea of surface forces and computing their value for each case.

In general, the stress at a boundary is given by

$$
R_{j}=n_{i} T_{i j}
$$

where $\vec{R}$ is the stress in the $\mathrm{j}$ direction in a plane with the normal vector $\hat{\mathrm{n}}$. T (all tensors will be shown in boldface) is thus defined as the stress tensor. $\mathbf{T}$ is more qualitatively defined for newtonian fluids by breaking it up into two parts as

$$
\begin{aligned}
T_{i j} & =-p \delta_{i j}+\tau_{i j}, \\
\tau_{i j} & =\mu\left(\frac{\partial v_{i}}{\partial x_{j}}+\frac{\partial v_{j}}{\partial x_{i}}\right) .
\end{aligned}
$$

Here $\tau$ is the viscous stress tensor for an incompressible fluid and $\mu$ is the dynamic viscosity $(\mu=\nu \rho)$. By equating the stress at the surface to the momentum flux deposited by the particles, it should be possible to model a fluid flow under the action of an energetic particle flux incident on the surface. By setting the momentum flux equal to zero, this surface boundary condition simplifies and results in the special case of no incident particle flux. It is worthwhile to do this case first 
in order to gain experience, and insight into approximations that may be helpful later.

\subsubsection{Zero particle flux}

Since the flow is steady state, fully developed and there is no momentum flux on the free surface to initiate or sustain lateral motion of the fluid (for a level channel), both transverse and coplanar velocity components, $\mathrm{v}$ and $\mathrm{w}$, are zero. The magnetic field can be divided into the constant external field $\vec{B}^{(0)}$, and the internally induced field $\vec{B}^{(1)}$, so that $\vec{B}=\vec{B}^{(0)}+\vec{B}^{(1)}$.

In the system of equations $1-7, \vec{J}$ can be eliminated by substituting Ampere's Law (equation 5). In this way the conservation of current equation 3 is automatically satisfied since the divergence of a curl is identically zero. Taking the curl of Ohm's Law, equation 4, and substituting in Faraday's Law, equation 6, for the curl of $\vec{E}$ gives

$$
0=\nabla \times\left(\vec{V} \times\left[\vec{B}^{(0)}+\vec{B}^{(1)}\right]\right)-\frac{1}{\mu_{m} \sigma} \nabla \times \nabla \times \vec{B}^{(1)},
$$

or the alternate form

$$
0=\nabla \times\left(u \hat{x} \times\left[\vec{B}^{(0)}+\vec{B}^{(1)}\right]\right)+\frac{1}{\mu_{m} \sigma} \nabla^{2} \vec{B}^{(1)},
$$

after some vector manipulation, and the substitution of equation 7 and the definition of the fully developed velocity $\vec{V}$. This is known as the magnetic induction equation.

If the first term of this equation is expanded out in component form it is seen that

$$
\left(\nabla \times\left(u \hat{x} \times\left[\vec{B}^{(0)}+\vec{B}^{(1)}\right]\right)\right)_{x}=\frac{\partial}{\partial y}\left(u\left[B_{y}^{(0)}+B_{y}^{(1)}\right]\right)+\frac{\partial}{\partial z}\left(u\left[B_{z}^{(0)}+B_{z}^{(0)}\right] g^{13}\right)
$$




$$
\left(\nabla \times\left(u \hat{x} \times\left[\vec{B}^{(0)}+\vec{B}^{(1)}\right]\right)\right)_{y}=\left(\nabla \times\left(u \hat{x} \times\left[\vec{B}^{(0)}+(\vec{B})^{1}\right]\right)\right)_{z}=0 .
$$

Dividing the magnetic induction equation 12 into components and substituting equation 14 gives the result that $\nabla^{2} B_{y}^{(1)}=\nabla^{2} B_{z}^{(1)}=0$. It can be shown that this leads to $B_{y}^{(1)}=B_{z}^{(1)}=0$. Using this result, the magnetic field and the new form of the magnetic induction equation can be written down as

$$
\begin{aligned}
\vec{B} & =\left(B_{x}^{(1)}, B_{y}^{(0)}, B_{z}^{(0)}\right) \\
0 & =\frac{\partial^{2} B_{x}^{(1)}}{\partial y^{2}}+\frac{\partial^{2} B_{x}^{(1)}}{\partial z^{2}}+\sigma \mu_{m}\left(B_{y}^{(0)} \frac{\partial u}{\partial y}+B_{z}^{(0)} \frac{\partial u}{\partial z}\right) .
\end{aligned}
$$

Equation 16 is the simplification of the magnetic induction equation for this particular problem. It is the dimensional version of the first equation (out of three) of model-1, the no particle flux case. The definition of $\vec{B}$, equation 15 , will be useful in deriving the second model equation from the Navier-Stokes equation of motion.

The steady state, fully developed version of equation 1 can be writen as

$$
0=-\frac{1}{\rho} \nabla p+\nu \nabla^{2} u \hat{x}+\frac{\vec{J} \times \vec{B}}{\rho}-g \cos \theta \hat{y}+g \sin \theta \hat{x}
$$

where $\theta$ is the angle of the acceleration of gravity to $\hat{x}$. As said before, Ampere's law, equation 5 , is used to eliminate the current. This, together with equation 15 allows $\vec{J}$ to be expressed in terms of $\vec{B}$.

$$
\vec{J}=\frac{1}{\mu_{m}}\left(0, \frac{\partial B_{x}^{(1)}}{\partial z},-\frac{\partial B_{x}^{(1)}}{\partial y}\right)
$$

Using this form of $\vec{J}$ and again equation 15 , the magnetic force term can be written as

$$
\frac{\vec{J} \times \vec{B}}{\rho}=\frac{1}{\rho \mu_{m}}\left(\left[B_{y}^{(0)} \frac{\partial B_{x}^{(1)}}{\partial y}+B_{z}^{(0)} \frac{\partial B_{x}^{(1)}}{\partial z}\right],-B_{x}^{(1)} \frac{\partial B_{x}^{(1)}}{\partial y},-B_{x}^{(1)} \frac{\partial B_{x}^{(1)}}{\partial z}\right)
$$


which can be substituted into the equation of motion to give

$$
\begin{aligned}
0= & -\frac{1}{\rho} \nabla\left(p+\frac{B_{x}^{(1)^{2}}}{2 \mu_{m}}\right)+\nu \nabla^{2} u \hat{x} \\
& +\frac{1}{\rho \mu_{m}}\left(B_{y}^{(0)} \frac{\partial B_{x}^{(1)}}{\partial y}+B_{z}^{(0)} \frac{\partial B_{x}^{(1)}}{\partial z}\right) \hat{x}-g \cos \theta \hat{y}+g \sin \theta \hat{x} .
\end{aligned}
$$

The term $p+\frac{B_{r}^{(1)^{2}}}{2 \mu_{m}}$ is the total pressure $p_{t}$, the sum of the mechanical and magnetic pressures. Since no other terms in expression 20 depends on $x$, then the gradient of $p_{t}$ in the $\hat{\mathrm{x}}$ direction can only be a constant, $\mathrm{k}$. This constant can be determined by looking at the $y$-component of equation 20 .

$$
0=-\frac{1}{\rho} \frac{\partial}{\partial y}\left(p+\frac{B_{x}^{(1)^{2}}}{2 \mu_{m}}\right)-g \cos \theta
$$

Integrating this expression from $y$ to $h_{f}$ gives the total pressure as

$$
p_{t}=g \rho \cos \theta\left(h_{f}-y\right)+p\left(h_{f}\right)
$$

where $p\left(h_{f}\right)$ is the pressure at the free surface. For the fully developed case, $h_{f}$ and $p\left(h_{f}\right)$ are not functions of $\mathrm{x}$. Therefore $\mathrm{k}$, the $\hat{\mathrm{x}}$ gradient of $p_{t}$, is zero. Thus this gradient can be dropped from the $\hat{x}$ equation of motion and its final form is written as

$$
0=\nu\left(\frac{\partial^{2} u}{\partial y^{2}}+\frac{\partial^{2} u}{\partial z^{2}}\right)+g \sin \theta+\frac{1}{\rho \mu_{m}}\left(B_{z}^{(0)} \frac{\partial B_{x}^{(1)}}{\partial z}+B_{y}^{(0)} \frac{\partial B_{x}^{(1)}}{\partial y}\right) .
$$

Equations 23 and 16 constitute a system of equations for the fully developed, steady state induced magnetic field and velocity profiles in terms of the $y$ and $z$ coordinates. If the fully developed (equilibrium) film height is known a priori, then this system with appropriate boundary conditions are sufficient for the determination of field and velocity. However this is not generally the case and another equation is necessary to close the system. The constant flowrate condition, 
which states that at any point along the duct the mass flowrate (and volumetric flowrate for an incompressible fluid) across a cross section is the same as at any other location, fulfills this purpose.

$$
Q_{0}=\int_{-b / 2}^{b / 2} \int_{0}^{h_{f}} u d z d y
$$

The volumetric flowrate, $Q_{o}$, is given as an initial condition and is preserved along the entire length of the flow. If, however, a specific equilibrium height is chosen, the system can be solved and the flowrate which yields such a $h_{f}$ can be determined from equation 24 .

The general system can then be normalized with the following non-dimensional parameters.

$$
\begin{aligned}
z^{*} & \equiv \frac{z}{b / 2} \\
y^{*} & \equiv \frac{y}{h} \\
B^{*} & \equiv \frac{B_{x}^{(1)}}{B_{z}^{(0)}} \\
u^{*} & \equiv \frac{u}{u_{o}} \\
Q_{o}^{*} & \equiv \frac{Q_{o}}{u_{o}(b / 2) h} \\
\tan \alpha & \equiv \frac{B_{y}^{(0)}}{B_{z}^{(0)}} \\
\beta & \equiv \frac{h_{f}}{b / 2} \\
F r & \equiv \frac{\left(u_{o}\right)^{2}}{g(b / 2)} \\
R e & \equiv \frac{u_{o}(b / 2)}{\nu} \\
R e_{m} & \equiv u_{o} \sigma \mu_{m}(b / 2) \\
H a & \equiv B_{z}^{(0)}(b / 2) \sqrt{\sigma / \rho \nu}
\end{aligned}
$$


Here $\alpha$ is the angle of the magnetic field to $\hat{z}$, and $u_{o}$ is the characteristic velocity. The abbreviations $F r, R e, R e_{m}$, and $H a$ are respectively the Froude number, Reynold's number, Magnetic Reynold's number, and Hartmann number. With these substitutions the non-dimensionalized system is now constructed.

$$
\begin{gathered}
\frac{\partial^{2} u^{*}}{\partial y^{* 2}}+\beta^{2} \frac{\partial^{2} u^{*}}{\partial z^{* 2}}+\frac{H a^{2} \beta^{2}}{R e_{m}}\left(\frac{\partial B^{*}}{\partial z^{*}}+\frac{\tan \alpha}{\beta} \frac{\partial B^{*}}{\partial y^{*}}\right)=\frac{R e \beta^{2}}{F r} \sin \theta \\
\frac{\partial^{2} B^{*}}{\partial y^{* 2}}+\beta^{2} \frac{\partial^{2} B^{*}}{\partial z^{* 2}}+R e_{m} \beta^{2}\left(\frac{\partial u^{*}}{\partial z^{*}}+\frac{\tan \alpha}{\beta} \frac{\partial u^{*}}{\partial y^{*}}\right)=0 \\
\int_{-1}^{1} \int_{0}^{1} u^{*} d y^{*} d z^{*}=Q_{o}^{*}
\end{gathered}
$$

The aspect ratio, $\beta^{-1}$, is very large for channel widths on the order of meters and film heights on the order of millimeters. Therefore, terms multiplied by $\beta$ and $\beta^{2}$ have a lesser effect on the bulk film flow. However, both Ha and Re can be very large and offset the small $\beta$ in terms where both are present.

With the equations derived, it becomes necessary to establish the boundary conditions for the velocity and induced magnetic field. As mentioned before, for no incident momentum flux the shear stress at the surface is zero. Also remember that the surface is flat and there is no $\mathrm{v}$ or $\mathrm{w}$ velocity. For this special case the shear stress condition is $\left.\frac{\partial u^{*}}{\partial y^{*}}\right|_{y^{*}=1}=0$. The no slip condition on the three channel walls gives $\left.u^{*}\right|_{z^{*}= \pm 1}=\left.u^{*}\right|_{y^{*}=0}=0$, which takes care of the velocity.

The magnetic field conditions are derived from the better known conditions for the electric current. The normal current at the free surface is zero, i. e. no current is leaving the film. This is also the case at the boundary between the channel wall and the outside world. This translates for the magnetic field into $\frac{\partial B^{*}}{\partial s}=0$ ( $\mathrm{s}$ is coordinate tangential to the boundary), or more simply $B^{*}=0$ since $s$ traces a closed loop. At the interior boundary between the fluid and the channel wall the normal current is conserved and the tangential currents are discontinuous 
according to the ratio $\left(J_{w}\right)_{g} /\left(J_{f}\right)_{s}=\sigma_{w} / \sigma_{f}$. Converting this to induced magnetic field conditions gives $B^{*}$ as continuous (provided that $\mu_{m}$ is the same for both materials), and $\frac{1}{\sigma_{w}} \frac{\partial B_{u}^{*}}{\partial n}=\frac{1}{\sigma_{f}} \frac{\partial B_{i}^{*}}{\partial n}$ where $\hat{\mathrm{n}}$ is the coordinate directed normal to the boundary. These conditions are sufficient for the induced field provided that this field is also solved for in the channel walls. The equation describing the field in the wall is the stationary analog of the magnetic induction equation 26.

$$
\frac{\partial^{2} B^{\star}}{\partial y^{\star 2}}+\beta^{2} \frac{\partial^{2} B^{\star}}{\partial z^{\star 2}}=0
$$

These equations: $25,26,27$, and 28 , and the boundary conditions given above are the complete zero particle flux version of model-1.

In order to avoid solving for the induced field in the channel walls it is convenient to introduce the thin wall boundary condition [38]. This condition assumes that the walls are thin enough that the effect of the current crossing the walls is negligible compared to the current flowing along the wall. The mathematical form of this approximation is derived from conservation of current at the boundary.

$$
\frac{\partial\left(J_{w}\right)_{s}}{\partial s}=-\frac{\left(J_{f}\right)_{n}}{t}
$$

Notice that this is a dimensional equation where $t$ is defined as the thickness of the wall. By substituting for the current in terms of the induced field in the fluid only, a self consistent condition can be constructed for the induced field in the fluid at the channel wall boundary, eliminating the need to solve for the field in the wall. Thus the induced field boundary conditions on the three walls are obtained and non-dimensionalized to give

$$
\begin{aligned}
& 0=\left(\Phi_{s} \frac{\partial B^{\star}}{\partial z^{\star}} \pm B^{\star}\right)_{z^{*}= \pm 1} \\
& 0=\left(\Phi_{b} \frac{\partial B^{\star}}{\partial y^{\star}}-\beta B^{*}\right)_{y^{*}=0},
\end{aligned}
$$


where $\Phi_{(s, b)}=\frac{\sigma_{(s, b)^{\ell}(s, b)}}{\sigma_{J}(b / 2)}$ is defined as the wall conductance ratio for the side or bottom $(\mathrm{s}, \mathrm{b})$ wall respectively. At the free surface the $B^{*}=0$ condition still holds.

The solution of this system will yield the velocity and induced field profiles, from which can be obtained the potential and current density as well. Wall conductances can be varied so that their effect on the film can be observed. Determination of the fully developed film height for a given set of flow parameters (i. e. substrate angle, channel width, and flowrate) is also possible. This will provide valuable design data since it is desirable to flow the film close to equilibrium in order to prevent fluctuations along the channel.

All of this data can be used to verify or discredit assumptions used in previous models, as well as compared to other results in order to judge their accuracy. With respect to the former, it will be possible to determine if the proceedure of integrating along the coplanar field direction using an assumed Hartmann profile, used in many of the previous developing film models $[30,14,32,33]$, is a valid one, or instead leads to erronous conclusion about the behavior of the film. Work towards the solution of this system is underway and preliminary results are already being produced.

\subsubsection{Non-zero particle flux}

Figure 9 depicts the geometry for the case where a particle flux from the plasma is incident on the free surface. The thickness of the plasma sheath [37] here is greatly exaggerated in order to show its effect on the particles. The ions are accelerated from the scrape-off layer (SOL), through the sheath, and strike the surface at a high speed. Also present is an elecrostatic force characterized by the tendancy 


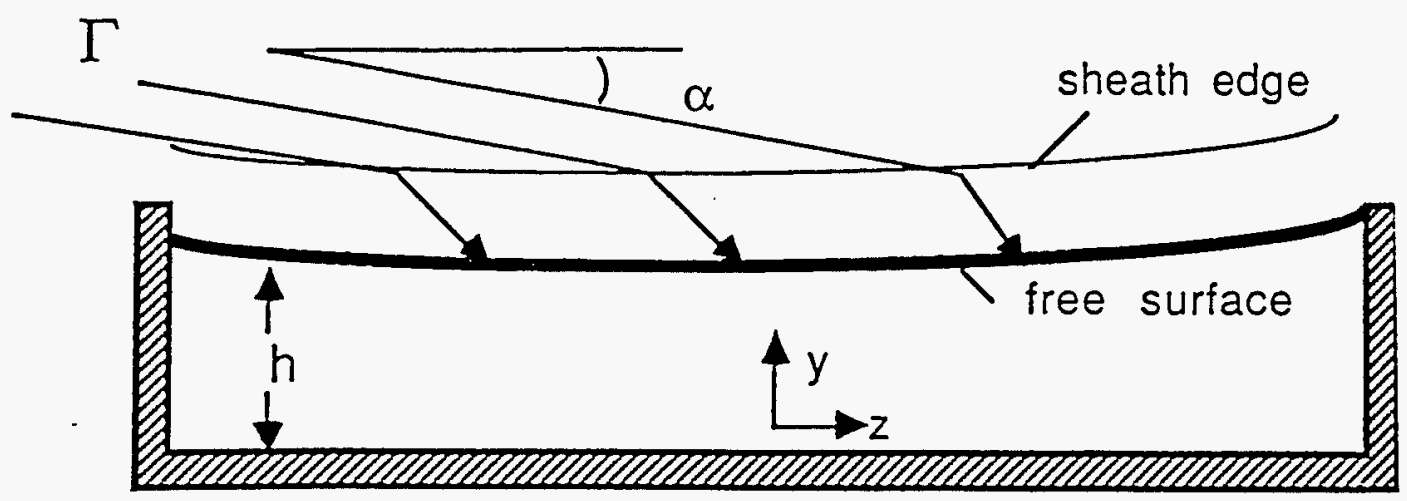

b

Figure 9. Film geometry with incident particle flux

of the positively charged plasma to pull on the negatively charged liquid surface. It seems likely, in order for momentum to be conserved in the sheath, that the additional force exerted by the accelerated ions pushing on the surface will be closely balanced by the electrostatic force pulling the surface. This assumption is corraborated by Liao [32] where both forces are oppositely directed and found to equal $2 n_{s} K T, n_{s}$ and $K$ are the particle density at the sheath edge and the Boltzmann constant. Thus the effect of the particles can be approximated as the ion momentum flux at the sheath edge, since the opposing effects of the sheath tend to cancel out.

The momentum flux can be calculated as

$$
\vec{\Gamma}_{p}=(M v) n_{s} \vec{v}
$$

where the subscript $\mathrm{p}$ denotes momentum; and $M, n_{s}$, and $v$ are the plasma particles' mass, number density and velocity at the sheath edge. Using ITER SOL data from Cohen [39], the magnitude of the momentum flux is estimated and given in Table 1. This is the momentum directed along the magnetic field 
Table 1. SOL plasma parameters and resulting momentum flux

\begin{tabular}{l|r}
\hline & DN-outboard \\
\hline$T_{\text {eo }}(\mathrm{eV})$ & 12.5 \\
$T_{i 0}(\mathrm{eV})$ & 10.2 \\
$q_{i}\left(\frac{\mathrm{NII}}{\mathrm{m}^{2}}\right)$ & 2.0 \\
$\Gamma_{p}\left(\frac{N}{\mathrm{~m}^{2}}\right)$ & 53.5 \\
\hline
\end{tabular}

line, at angle $\alpha$ to $\hat{z}$. The momentum in the plane perpendicular to the field line (due to thermal gyro-motion) can be averaged to give an added normal component, but this will be disregarded here due to its small magnitude and complexity of computation. These SOL parameters are the values at the point of maximum heat deposition on the divertor plate. The column DN-outboard stands for a double null, outer divertor configuration.

The relative size of this momentum flux (surface force density) can be compared to the hydrostatic pressure required to raise the fluid a typical scale length. In the transverse direction this scale length is on the order of millimeters.

\begin{tabular}{l|r} 
metal & $\rho g h$ \\
\hline Gallium & 60.8 \\
Lithium & 4.9 \\
Pb-Bi-Sn-Cd-In & 89.9
\end{tabular}

(These estimates are using $h=1 \mathrm{~mm}$. Information on the $\mathrm{Pb}-\mathrm{Bi}-\ldots$ eutectic relevancy is given in Chapter 4.) When this pressure is compared to the magnitude of the momentum flux, it is obvious that the particles can significantly affect the film, especially for lithium. This effect, however, is dependant on the angle of incidence and the rate of change of the pressure [32] over the length or width of the duct. 
The difficulty involved in computing the velocity and film height for this case stems from the introduction of a non-uniform film height and complicated velocity boundary conditions at the free surface. As mentioned before, the approach desired here is to equate the stress at the boundary with momentum flux from the particles. This momentum, as shown above, will in general have both normal and tangential components, thus affecting both the shear stress and the pressure conditions at the surface.

In order to find the form of the free surface, it will be necessary to solve the momentum equation. The $\hat{y}$ and $\hat{z}$ components of the Navier-Stokes equation for a film fully developed in $\hat{x}$ are given below.

$$
\begin{aligned}
\frac{\partial v}{\partial t}+v \frac{\partial v}{\partial y}+w \frac{\partial v}{\partial z} & =-\frac{1}{\rho} \frac{\partial p}{\partial y}+\nu\left(\frac{\partial^{2} v}{\partial y^{2}}+\frac{\partial^{2} v}{\partial z^{2}}\right)+\frac{(\vec{J} \times \vec{B})_{y}}{\rho}-g_{y} \\
\frac{\partial w}{\partial t}+v \frac{\partial w}{\partial y}+w \frac{\partial w}{\partial z} & =-\frac{1}{\rho} \frac{\partial p}{\partial z}+\nu\left(\frac{\partial^{2} w}{\partial y^{2}}+\frac{\partial^{2} w}{\partial z^{2}}\right)+\frac{(\vec{J} \times \vec{B})_{z}}{\rho}
\end{aligned}
$$

These equations are not dependent on $u$ and can be solved, along with the mass continuity and electromagnetic equations (see equations $2-7$ ) for the circulating flow in a yz cross-section.

The boundary conditions on the three channel walls are the same no-slip conditions used for the no particle flux case. On the free surface, however, things get more complicated. If the angle of the free surface to $\hat{z}$ is defined as $\gamma$, then $\tan \gamma=\frac{d h}{d z}$. The unit normal to the free surface is $\hat{n}=(0, \zeta(z), \eta(z))$, where

$$
\begin{aligned}
& \zeta(z)=\cos \gamma=\frac{1}{\sqrt{\frac{d h^{2}}{d z}+1}} \\
& \eta(z)=-\sin \gamma=\frac{-\frac{d h}{d z}}{\sqrt{\frac{d h^{2}}{d z}+1}}
\end{aligned}
$$


Using the above definitions, the stress conditions are constructed from equations 8,9 , and 10 .

$$
\begin{aligned}
\zeta\left[-p+2 \mu \frac{\partial v}{\partial y}\right]+\eta \mu\left[\frac{\partial v}{\partial z}+\frac{\partial w}{\partial y}\right] & =\left(\Gamma_{p}\right)_{y} \\
\eta\left[-p+2 \mu \frac{\partial w}{\partial z}\right]+\zeta \mu\left[\frac{\partial v}{\partial z}+\frac{\partial w}{\partial y}\right] & =\left(\Gamma_{p}\right)_{z}
\end{aligned}
$$

The value of $\left(\Gamma_{p}\right)_{y, z}$ will be determined from the angle $\alpha$ and the magnitude given in Table 1.

It is currently planned to solve the system of equations using a version of the hydrodynamic program SOLA-VOF [40]. This program was written to solve twodimensional fluid flow problems with multiple free boundaries. Modifications of the surface boundary conditions to account for the momentum flux should not prove too difficult. However, incorporating MHD forces may prove to be quite a task. As an initial problem, the solution for the free surface and velocity profiles will be obtained in the non-magnetic case.

With model-1 complete we will be in a position to evaluate the relative effects of sidewall and substrate conductivity in a channel with large aspect ratio, as mentioned in the no particle flux case, and the influence of the plasma momentum. Should the momentum flux prove to exert a significant effect, the idea of integrating over the channel width may have to be seriously altered or scraped all together in the construction of developing film models.

\subsection{Model-2: developing film}

As indicated in the Introduction, model-2 will describe a developing liquid metal film under the influence of space and time varying magnetic fields. Since estimates 
of the equilibrium height in past models [33] determine it to be a function of the field, it is only logical to assume that changing fields will result in changing film heights. Electric fields induced by a time varying magnetic field will also induce currents that affect the film behavior. Prediction of this phenomena is especially relevant to determining the survivability of the film in the case of a plasma disruption.

Like model-1, the developing film case will involve equations derived from the MHD equations given at the beginning of this chapter (see page 27). The particular assumptions that will be used cannot yet be given as they will most likely depend on the model-1 results. Ideally it would be nice to derive a model that performs a three-dimensional analysis incorporating both sidewalls and plasma wind as well as varying magnetic fields. This much work may not be necessary if the effect of the plasma wind is insignificant enough to allow averaging over the film width with the modified profiles determined by the model-1, no particle flux . case.

With the completed model, investigation of film flows in magnetic field configurations that will likely be encountered in a tokamak device can be performed. These configuration include, among others, a $1 / x$ dependance of the main coplanar field, rapidly time varying transverse fields, and sign inverting transverse: fields. Conditions on these fields in conjunction with other flow parameters will be established to show the parameter space allowing a usable film to exist. 


\section{Chapter 4}

\section{Experiment}

Proposed in conjunction with the mathematical models is the construction of an experimental facility and performance of free surface testing. The purpose of preliminary experiments will be to investigate the feasibility of creating a LM thin film flow in the presence of a magnetic field. More quantitative measurements of the film height, electric potential, and eventually the velocity profile are possible as more diagnostics are added to the original system. Thus the experiment will be useful in benchmarking the mathematical models, validating the approximations used and pointing out the need for more detailed mathematical efforts.

\subsection{Construction of experimental facility}

The facility in which these proposed experiments will be performed must be constructed. For an LM-MHD experiment, two separate systems must be built, the 
magnetic system, and the LM circulating loop. These systems will be built independently of each other and then combined for the experiment itself. The completed set-up will be known as the UCLA-MEGA Loop Facility where MEGA is an acronym that stands for ME-tal G-oes A-round. This particular experiment is the MHD-Film Flow Experiment (MHD-FFX). A basic diagram with preliminary dimensions of MEGA is shown in Figure 10.

\subsubsection{Magnetic field system}

The magnetic system consists of four circular electromagnet coils mounted on a rack with a common axis, a coil power supply, and a water coolant supply for circulation in the coils. The coils were originally part of the TARA Mirror Experiment at the Massachusetts Institute of Technology and were loaned to UCLA for use in MEGA. Given in Table 2 are the physical specifications of the coils. The coolant flowrate and pressure quoted here are for Tara operating conditions and will be modified as necessary for this experiment. The physical geometry of an individual coils is represented pictorially in Figure 11.

Calculated from this data is the distribution of the magnetic field (in Tesla per 1000 Amps) and lines of force, which are shown in Figure 12 and 13. The crossed lines on the plots represent the proposed size of the film flow in the interior of the

coil array. The maximum ripple $\left(B_{\text {radial }} / B_{\text {total }}\right)$ in the interior can be decreased by moving the coils closer together, although this also decreases the working volume. The maximum field magnitude will be controlled by the amount of current that can be supplied. The limit on the current for steady state operation of the coils will be determined by the coolant capability.

Preliminary heat transfer calculations have been performed. For the coolant 


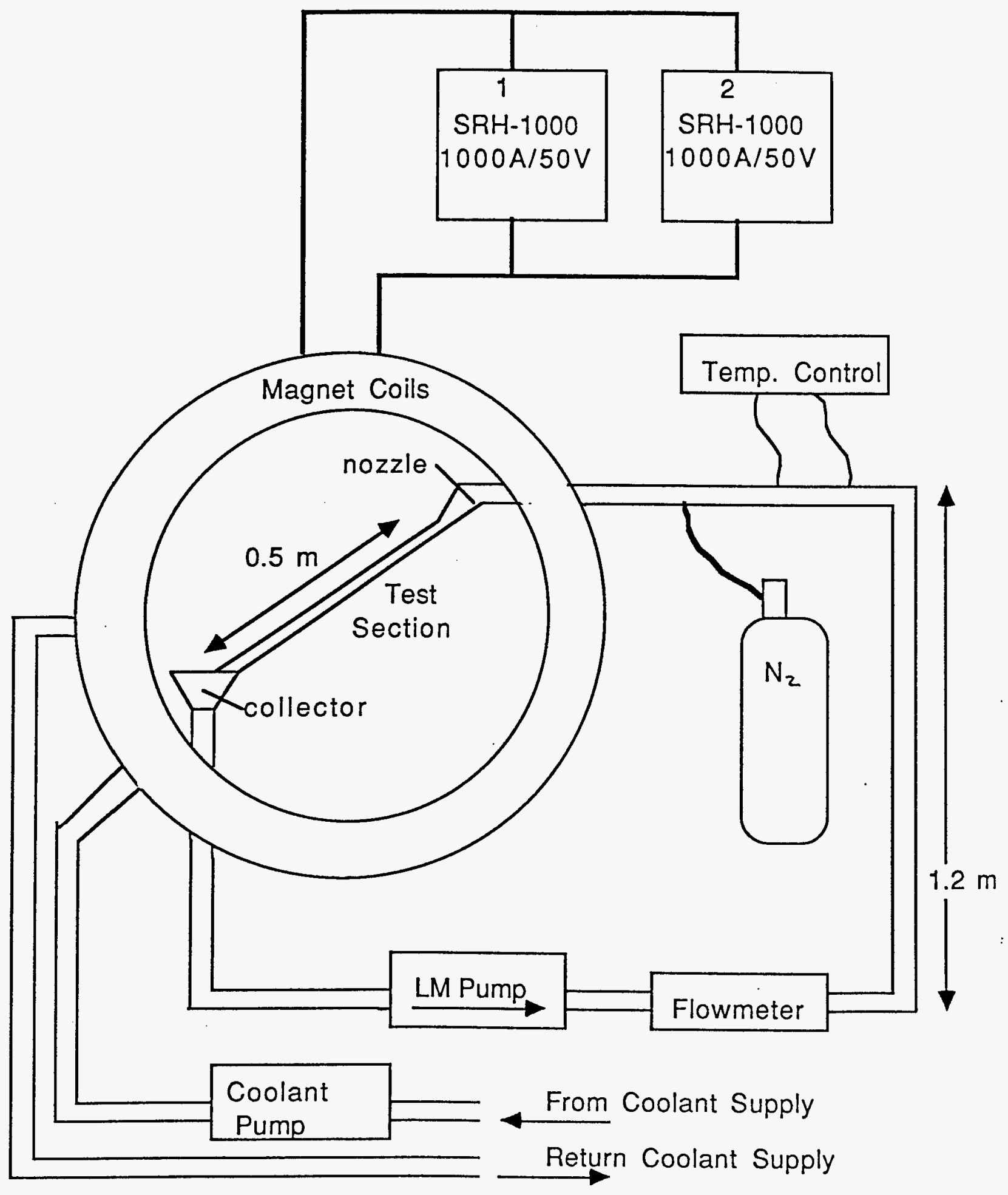

Figure 10. UCLA-MEGA loop diagram 
Table 2. Magnet coil physical specifications

\begin{tabular}{l|rl}
\hline & & \\
Number of Coils & 4 & \\
Coil Resistance $\left(20^{\circ}\right)$ & 7.23 & $\mathrm{~m} \Omega$ \\
Max. Current (steady state) & 1200 & $\mathrm{~A}$ \\
Voltage per Coil & 10 & $\mathrm{~V}$ \\
Double Layer Pancakes/Coil & 2 & \\
Turns per Layer & 7 & \\
Total Turns per Coil & 28 & \\
Coil Thickness & 7.2 & $\mathrm{~cm}$ \\
Coil Inner Diameter & 78.59 & $\mathrm{~cm}$ \\
Coil Outer Diameter & 101.75 & $\mathrm{~cm}$ \\
Coil Mean Turn Length & 281.94 & $\mathrm{~cm}$ \\
Conductor Length per pancake & 40.23 & $\mathrm{~m}$ \\
Coolant Differential Pressure & 690 & $\mathrm{kPa}$ \\
Coolant Flowrate & 0.1628 & $\mathrm{~L} / \mathrm{s}$ \\
& & \\
Conductor Dimensions & & \\
width/height & 1.499 & $\mathrm{~cm}$ \\
coolant hole diameter & 0.635 & $\mathrm{~cm}$ \\
corner radius & 0.127 & $\mathrm{~cm}$ \\
\hline \multicolumn{2}{|l}{}
\end{tabular}




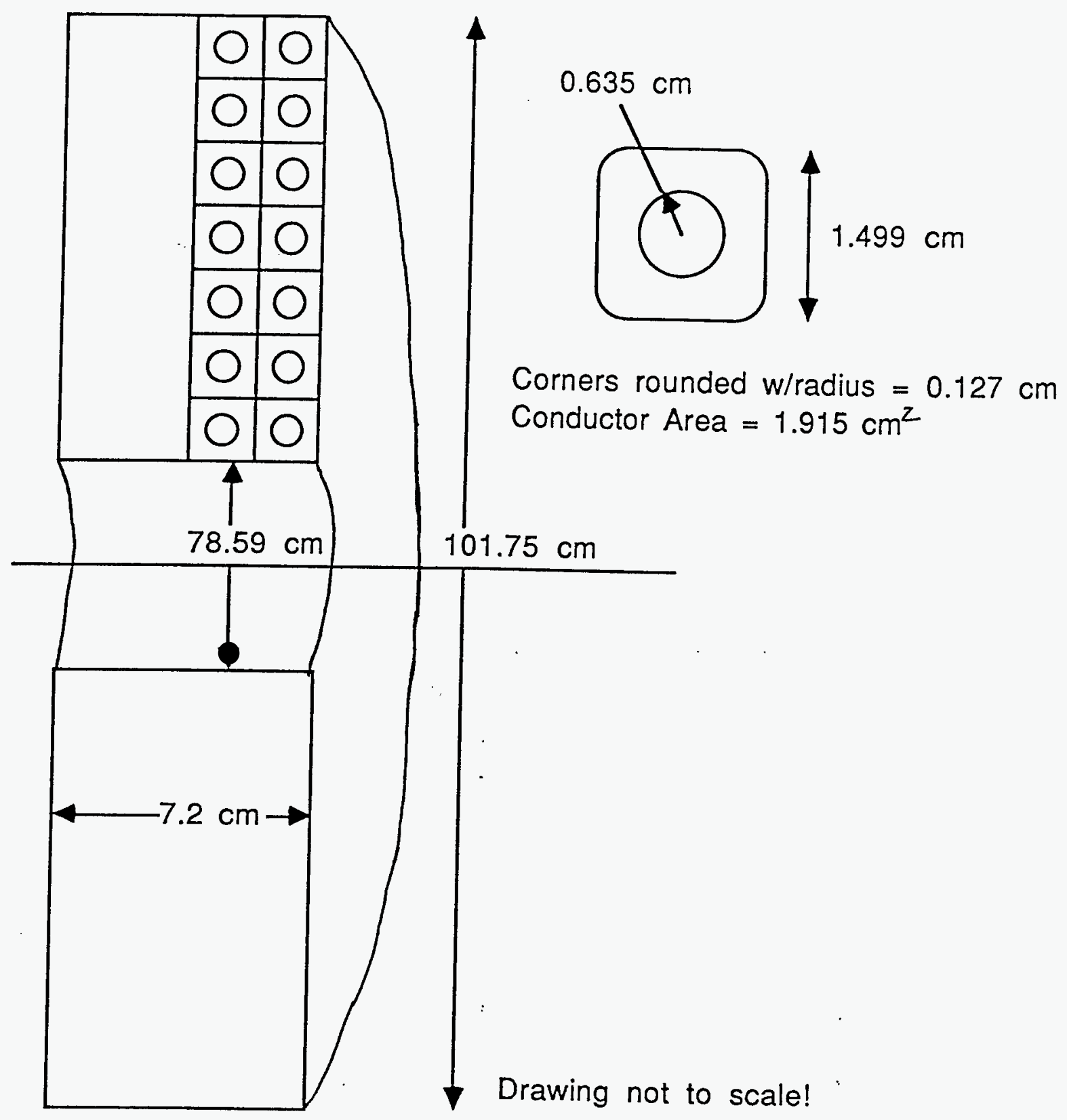

Figure 11. Magnetic coils cutaway view 



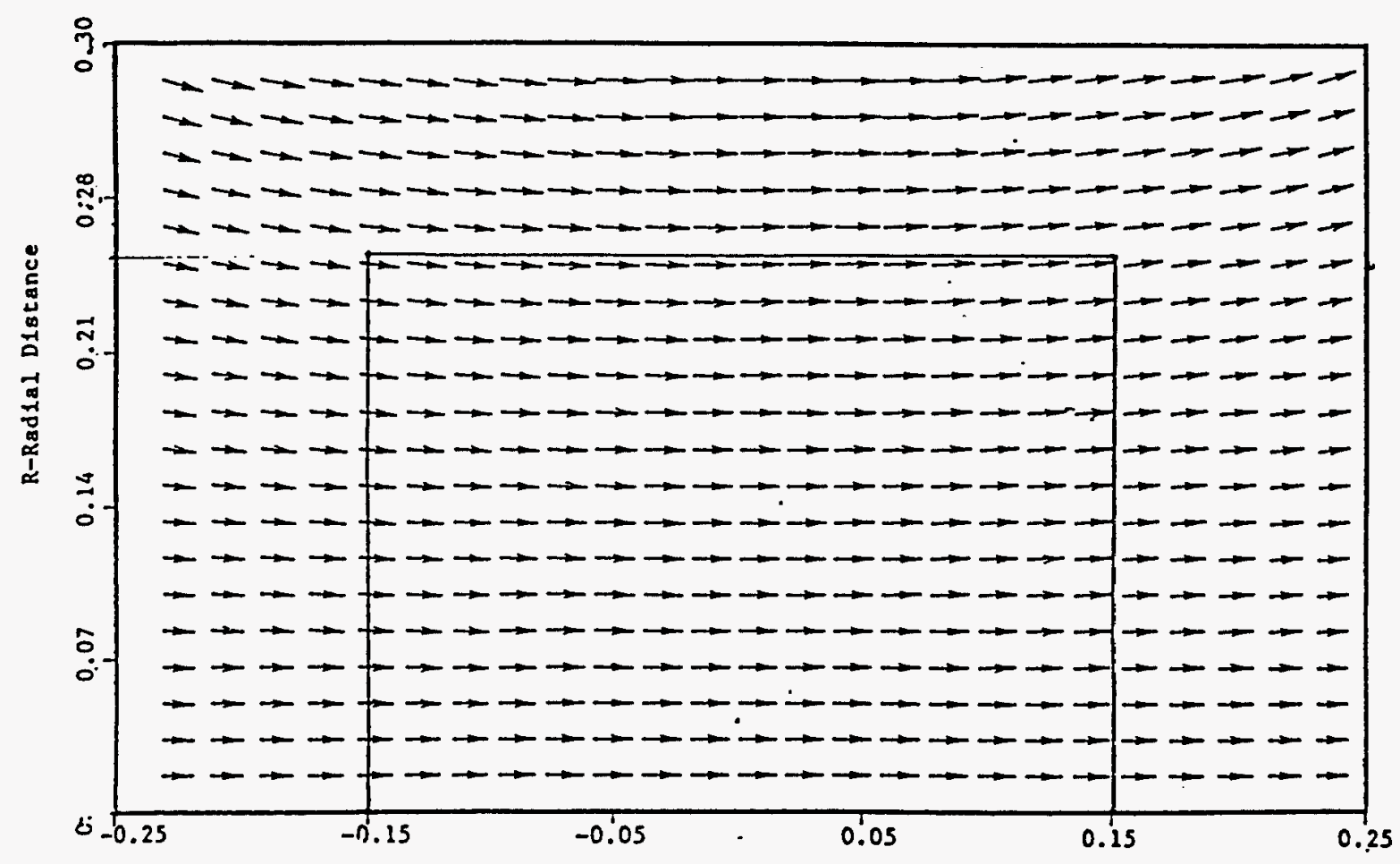

Figure 13. 4-coil set lines of force (6 in. spacing)

flowrate given in Table 2, a bulk coolant temperature rise at the outlet of about $5.3^{\circ} \mathrm{C}$ for $1000 \mathrm{~A}$ of current per pancake is predicted. This figure will increase quadratically with current. The peak temperature rise at the outlet is less than a degree higher than the bulk (for $1000 \mathrm{~A}$ ). Based on these calculations the operating current could go as high as $3-4 \mathrm{kA}$. More detailed calculations and experiments will be performed in order to more quantitatively determine the operating limits of the magnets.

The current will be supplied from a series of arc welder power supplies also loaned from MIT. The supplies are rated for $1000 \mathrm{~A}$ at $50 \mathrm{~V}$ and will be used in parallel to reach higher currents. 


\subsubsection{LM circulation system}

The liquid metal circulation system is made up of a pump and flowmeter, supply and return piping, and a test section residing inside the coil interior. Other elements of the LM system include: heaters, depending on the melting temperature of the LM; a storage tank, for storage of the metal for long periods of down time; and an alternate atmosphere or vacuum system, to prevent LM contact with oxygen or water. The need for these other system components are somewhat determined by the properties of the LM used in the loop.

For MHD-FFX it is desirable to have a LM that is lightweight, non-reactive with structural material, air, and water, wettable to the channel material, inexpensive, liquid at low temperatures, and highly interactive with a magnetic field. Unfortunately, no metal satisfying all these conditions has been found. The three main choices are Gallium (or a Ga-Eutectic), Sodium-Potassium (NaK), or a Lead-Bismuth Eutectic (Pb-Bi).

Gallium is also one of the choices that was given for the reactor LM. For this reason it would be nice to use $\mathrm{Ga}$ so that problems with wettability and structural corrosion that might be faced in a reactor situation could also be explored. However, $\mathrm{Ga}$ is far too expensive for this scale of experiment and must be discarded for this reason unless a less expensive source of $\mathrm{Ga}$ is found.

$\mathrm{NaK}$ has many desirable physical characteristics. It is very light relative to $\mathrm{Ga}$ and $\mathrm{Pb}-\mathrm{Bi}$ and is therefore easier to pump to higher velocities typical of a fusion device. It has a high interaction with the magnetic field making it possible to reach more fusion relevant similarity parameter ranges with the smaller magnetic field of our magnets. $\mathrm{NaK}$ has a melting temperature of less than $0^{\circ} \mathrm{C}$ so that it is always in liquid form and never requires heating. And also, it is less expensive 
Table 3. $\mathrm{Pb}-\mathrm{Bi}-\mathrm{Sn}$-Cd-In physical properties

\begin{tabular}{l|r}
\hline Melting Point $(C)$ & 47 \\
Kinematic Viscosity $\left(\mathrm{m}^{2} / \mathrm{s}\right)$ & $1.83 \times 10^{-7}$ \\
Mass Density $\left(\mathrm{kg} / \mathrm{m}^{3}\right)$ & 9160 \\
Electrical Conductivity $\left(\Omega^{-1} \mathrm{~m}^{-1}\right)$ & $1.96 \times 10^{6}$ \\
\hline
\end{tabular}

than the other option, $\mathrm{Pb}-\mathrm{Bi}$. However, $\mathrm{NaK}$ is extremely reactive with air and water and thus represents a safety hazard if not properly contained. Due to the fact that this experiment is the first effort in LM flow and is located very close to other experiments and personnel, inherent safety is of primary importance since mistakes are sure to be made in the construction and testing of this facility. It is possible that at a later time, $\mathrm{NaK}$ may be introduced to MEGA for the reasons given above, but only after all safety reservations have been satisfied.

This leaves only the $\mathrm{Pb}-\mathrm{Bi}$ Eutectic. The specific alloy chosen is $22.6 \%$ Lead, 44.7\% Bismuth, $8.3 \%$ Tin (Sn), 5.3\% Cadmium (Cd), and $19.1 \%$ Indium (In). The addition of In to this Wood's Metal eutectic lowers the melting point and improves wettability of $\mathrm{Pb}-\mathrm{Bi}$ alloys. The interaction of this metal with the magnetic field is slightly less than $\mathrm{Ga}$ and much less than $\mathrm{NaK}$ so similarity parameter will be low compared to a fusion environment. Its density is high making it the most difficult to pump and support properly. Poisoning by $\mathrm{Pb}$ or $\mathrm{Cd}$ fumes isn't expected to be a problem but due to the high oxidation rate of the metal, an alternate atmosphere $\left(N_{2}\right)$ will be provided anyway. The physical properties of this alloy are given in Table $3[41,42]$.

Calculations for the pressure drop and loop volume were performed based on a preliminary assessment of the dimensions of the loop and test section. An example case of a $0.3 \times 0.005 \mathrm{~m}$ film moving at $3 \mathrm{~m} / \mathrm{s}\left(Q=0.0045 \mathrm{~m}^{3} / \mathrm{s}\right)$ can be sustained 
by a $270 \mathrm{kPa}$ differential pressure with a loop volume of about $9.09 \mathrm{~L}$ of LM. This volume corresponds to about $84.4 \mathrm{~kg}$. For comparison sake it is worth noting that if $\mathrm{NaK}$ were used, this same flowrate and loop volume would correspond to mass of $10.5 \mathrm{~kg}$ and a pressure of $21.4 \mathrm{kPa}$, an order of magnitude lower.

\subsection{MHD-FFX design and test plan}

Specifics of the test section design have not yet been finally decided. It is currently planned to make two test, one thin $(\approx 9 \mathrm{~cm})$ in order to attempt to reproduce previous Soviet results, and one fairly wide with respect to previous experiments, from 0.3 to $0.5 \mathrm{~m}$. This large width is one of the unique features of MHDFFX. The wider the channel, though, the less uniform the magnetic field will be over the area of the film. This ripple, however, is characteristic of tokamak field distributions as well. In addition to specifying the film size, design of a film former and collector section is also required. A slot nozzle type arrangement with vanes to uniformly spread out the LM and a stabilization region before the opening will be the first attempt. Due to the large width, the design of the film former is of more importance than in smaller experiments. The collector and drain back to the pump should not be much of a problem. The walls of the channel will be thin copper and the $\mathrm{Pb}-\mathrm{Bi}$ should wet this surface after sufficient cleaning and exposure to the LM. It is suggested that a rough surface [7] is desirable since stagnant LM will fill in the crevices and better wet the moving film.

A outline of the MHD-FFX Test Plan is given below. There is no time scale imposed on each of the steps but each large roman numeral identifies groups of activities that will be performed concurrently. 


\section{MHD-FFX Test Plan}

I. Material Testing, Design, and Facility Construction

A. Construction of the MEGA Facility

1. Magnetic System

a. procurement of equipment

i. coolant pump (may not be needed with E-4 chilled water)

ii. piping

iii. gauss meter

iv. miscellaneous electrical and fluid flow diagnostics

b. retaping and cleaning coils and rack

c. repairing damaged coolant inlets

d. assembly of system

e. testing of magnetic system

i. coolant circulation testing

ii. low field magnet testing

iii. high field magnet testing

2. LM Circulation Loop

a. procurement of equipment

i. LM pump

ii. piping

iii. flowmeter/controller

iv. $\mathrm{Pb}-\mathrm{Bi}$

v. vacuum/alternate atmosphere equipment

b. assembly of system

c. testing of circulation system 
i. vacuum/alternate atmosphere test

ii. calibration of flowmeter

iii. verification of circulation

B. Material Test of Pb-Bi

1. Wetting to copper

a. effect of different cleaning fluxes

a. effect of heat and copper roughness

2. Determination of oxidation severity

3. Effect of expansion upon solidification on container

C. Design of MHD-FFX

1. Design of test section

2. Design of diagnostics

II. Final Assembly and Running the First Stage Experiment

A. Introduction of initial narrow test section into the loop

B. Without magnetic field testing

1. even flow filling the channel/performance of nozzle

2. wetting

3. collection of the LM

C. With magnetic field testing (same as above)

D. Visual investigation of performance

1. evenness of flow (turbulence)

2. performance of film former and collector

E. Measurement of height and potential distribution

1. effect of different flow angles and flowrates

2. behavior in a rippling magnetic field 
F. Repeat A - E for wide test section

III. Draw Conclusions and Retest when Necessary

The measurement of the film height and potential will require the development of diagnostics that can be controlled from outside the test section. Such instruments have been developed by Soviet and Japanese experimentalists [31, 43]. One such device is a capacitive probe that measures the capacitance between two "quarter-size" plates. From this is determined the average height over that area. This particular instrument is not as useful for measuring wave heights but has the desirable feature of no moving parts and can be remotely computer controlled. The other probe is a simple sliding, needle-like electrode that completes an electrical circuit or measures a potential difference to some reference when it contacts the surface. The sliding action would be manually performed in its simplest form. It is possible that both of these measuring devices will be used, but the simple sliding probe is more likely for this preliminary experiment.

The data from these measurements will be particularly valuable for the benchmarking of mathematical models, both the ones proposd in Chapter 3 and previous efforts described in Chapter 2. In addition to these results, practical engineering information regarding the performance of the film former and collector, and response of the fast film to channel imperfections will be gathered. This information will be useful in the construction of more fusion relevent experiments performed either at MEGA or elsewhere. 


\section{Chapter 5}

\section{Summary}

Due to the lifetime limitations predicted for solid materials used as a tokamak divertor, the idea of utilizing a film of liquid metal for protection of the surface has been advanced. Questions concerning the interaction of this conducting film with the magnetic field need to be addressed in order to access the feasibility and attractiveness of this scheme in comparison to other alternative ideas. Modelling the behavior of the film in a fusion environment is not easily accomplished. For this reason, preliminary models have used simplifications of the basic governing equations in order to approximate the film flow variables More complicated issues directly relevant to the fusion situation, though, have been left largely unexplored.

Proposed here are two mathematical models that address several key issues that may seriously influence the film flow. These issues include the effect of the plasma wind incident on the surface, of conducting sidewalls and substrate, and varying magnetic fields likely to occur in a reactor situation. These phenomena represent the major remaining MHD-related questions concerning the possibility of establishing and maintaining a film flow. Although even here these effects will 
not be determined in complete generality, it is felt that these models are the next logical step in order to establish the severity of their influence on the film. Once done, it will be possible to model many fusion-like situations and predict the film reaction. From this data tendencies of the film behavior will be established and conclusions drawn about the possibility of liquid metal film protection of divertor surfaces.

Also proposed is the building of an experimental facility, the UCLA-MEGA Loop, for the physical demonstration and measurement of LM-MHD thin film flow in a channel with a large aspect ratio $\left(\beta^{-1} \gg 1\right)$. Herein lies the difference between this experiment and others performed previously in the Soviet Union. The magnets in the MEGA-Loop have a large interior area and will allow wider thin films than previously possible in the higher field, but smaller gap area magnets. The initial experiment will include the demonstration of a wide film flow in a rippling magnetic field. Measurements of the film height and electric potential will then be taken for a variety of different flow parameters and used in the validation of the mathematical models.

Together, these two efforts represent a considerable advancement in understanding and predictive capabilities regarding LM-MHD thin film flows for fusion divertor/limiter protection. Based on the data produced from these models, LM protection can be evaluated form a much more fusion relevant standpoint.

\section{Acknowledgments}

A special thanks to Prof. Mohamed Abdou and Dr. Mark Tillack for their help in editting this document, and to Dr. Igor Evtushenko and Dr. Evgeni Murav'ev for many informative conversations. Thanks also to the Massachusetts Institute of 
Technology for their generous loan of the equipment for MEGA, especially Leslie Bromberg for facilitating the arrangements on that end and Julie Austin for all her help on my end. This and future work is partially supported by an ORAU/DOE Fusion Energy Technology Fellowship, for which I am most grateful. The paper was produced using the $\mathrm{IAT}_{\mathrm{E}} \mathrm{X}$ Document Preparation System [44]. Thanks for listening - NBM. 


\section{Bibliography}

[1] C. P. Liao, M. Kazimi, J. Meyer. On Liquid Metal Divertors. Submitted to: Fusion Technology. 1991.

[2] M. A. Abdou. INTOR Impurity Control and First Wall System. Proceedings of 12th Symposium of INTOR. Vol. I, pp. 39-56, 1982.

[3] E. V. Murav'ev, A. V. Kashirshij, P. V. Romanov, O. V. Belorusova, V. S. Petrov, et al. Development of a Liquid Metal Droplet Divertor System for ITER. US-USSR Collaboration on Liquid Metal Blanket and Divertor Problems, Argonne National Laboratory, Nov. 1990.

[4] I. R. Kirillov, I. V. Mazul, E. V. Murav'ev. Alternative Concepts of the Divertor Targets. Soviet Contribution to ITER Advanced Divertor Specialists' Meeting in Garching, Oct. 1990.

[5] A. M. Hassanein, D. L. Smith. Evaluation of Liquid Metal Protection of Limiter/Divertors in Fusion Reactors. 8th Topical Meeting of the Technology of Fusion Energy, Salt Lake City. Oct. 1988.

[6] E. V. Murav'ev editor. Liquid Metal Droplet Heat Removal System for the Divertor of a Tokamak Reactor. US-USSR Collaboration on Liquid Metal 
Blanket and Divertor Problems, Argonne National Laboratory, Argonne Nat. Lab. Nov. 1989.

[7] M. E. Lebedev, B. S. Fokin, V. V. Yakovlev. MHD Flow having a Free Surface on a Divertor Contact Assembly Model. Publishing Status Unknown.

[8] E. V. Murav'ev. Liquid Metal Devices for Systems of Impurity Control and First Wall Shielding in Tokamak Thermonuclear Reactors. All-Union Conference on Engineering Problems of Thermonuclear Reactors, USSR. Vol. 4, Jan. 1984. (ANL-TRANS-89-4)

[9] V. N. Dem'yanenko, B. G. Karasev, A. F. Koleshichenko, I. V. Lavrentev, O. A. Lielausis, et al. Liquid Metal in the Magnetic Field of a Tokamak Reactor. Magnetohydodynamics. No. 1, pp. 104-124, Jan-Mar. 1988.

[10] K. A. Werley. High Speed Beam of Lithium Droplets for Collecting Diverted Energy and Particles in ITER. IEEE 13th Symposium on Fusion Energy. Oct. 1989.

[11] E. V. Murav'ev. MHD Flow with a Free Surface in a Fusion Reactor with Magnetically Confined Plasma. 11th Riga Conference on MHD, Salaspils: Vol. 1, pp. 107-110, 1984.

[12] B. Badger, et al. Wisconsin Toroidal Fusion Reactor Design Study. UWFDM-68, Vol. 2, pp. III-D-1-5, 1974.

[13] USSR Contibutions to the 14th Session of INTOR Workshop. Phase 2-A, Viena. 1986. 
[14] N. B. Morley. Analysis of Thin Film Liquid Metal Protection of Fusion Reactor Plasma Contact Surfaces. UCLA Master's Thesis, UCLA-FNT-40. 1990.

[15] E. V. Murav'ev, V. A. Shmatenko, V. V. Yakovlev. Experimental Study of a Flow with a Free Surface in a Turn in a Strong Magnetic Field. Magnetohydodynamics. No. 1, pp. 103-106, Jan-Mar. 1987.

[16] E. V. Murav'ev, A. V. Tananaev, A. V. Chudov, V. V. Yakovlev. MHD Flow with a Free Surface Along the Substrate of a Liquid Metal Limiter: Experimental Results. 11th Riga Conference on MHD, Salaspils. Vol. 1, pp. 111-114, 1984.

[17] A. V. Tananaev, E. V. Murav'ev, V. V. Yakovlev. Investigation of Film MHD Flow as Applied to the Problem of Developing LM Diaphragms and Collector Devices of a Divertor in a Thermonuclear Tokamak Reactor. All-Union Conference on the Engineering Problems of Thermonuclear Reactors, USSR. Vol. 4, Jan. 1984. (ANL-TRANS-89-3)

[18] W. M. Wells. A System for Handling Divertor Ion and Energy Flux Based on a Lithium Droplet Cloud. Nuclear Tech/Fusion. Vol. 1, Jan. 1981.

[19] V. A. Bernshtam, A. B. Isers, S. V. Kozyrev, S. A. Lifits, A. I. El'kin. Deformation and Stability of Liquid Metal Contact Surfaces at the First Wall and Divertor of Tokamak Thermonuclear Reactors. All-Union Conference on Engineering Problems of Themonuclear Reactors, USSR. Vol. 4, Jan. 1984. (ANL-TRANS-89-2) 
[20] G. Gerbeth, M. Kaudze, A. Gailitis. Deformation of an Electrically Conducting Drop in a Magnetic Field. Liquid Metal Magnetohydrodynamics. Kluwer Academic Publishers, pp. 287-292, 1989.

[21] V. N. Dem'yanenko, V. O. Vodyanyak, A. F. Koleshichenko, E. V. Murav'ev. Shaping of Liquid Metal Flows with Predetermined Parameters in a Tokamak Magnetic Field. US-USSR Collaboration on Liquid Metal Blanket and Divertor Problems, Argonne National Laboratory, Nov. 1990.

[22] E. V. Murav'ev. Private Communications. Kurchatov Institute of Atomic Energy in Moscow, USSR. 1991.

[23] V. O. Vodyanyuk, V. N. Demyanenko, A. F. Kolesnichenko, S. V. Mirnov, E. V. Muravev, et al. Liquid Metal Tokamak Limiter: Statement of Problems and First Results. Soviet J. Plasma Phys. 14(5), May 1988.

[24] M. S. Tillack. Liquid Metal Primer for Fusion. to be published. 1990.

[25] R. Moreau. Magnetohydrodynamics. Kluwer Academic Publishers, 1990.

[26] R. A. Alpher, H. Hurwitz Jr., R. H. Johnson, D. R. White. Some Studies of Free Surface Mercury Magnetohydrodynamics. Rev. Modern Phys. Vol. 32(4), pp. 758-769, Oct. 1960.

[27] E. V. Muravev, V. V. Yakovlev. MHD Flow with a Free Surface Along the Wall of a Liquid Metal Limiter: 2. Calculational Model. 11th Riga Conference on MHD, Salaspils. Vol. 1, 1984. 
[28] T. N. Aitov, E. M. Kirillina. Flow of Electrically Conducting Liquid in a Thin Layer with a Free Surface Under the Action of a Strong Magnetic Field. Magnetohydrodynamics. No. 3. pp. 71-76, Jul-Sept. 1985.

[29] E. V. Murav'ev. Film MHD Flows Under Conditions of a Thermonuclear Reactor. Magnetohydrodynamics. No. 1, pp. 125-138, Jan-Mar. 1988.

[30] T. N. Aitov, A. B. Ivanov, A. V. Tananaev. Flow of Liquid Metal in a Chute in a Coplanar Magnetic Field. Magnetohydrodynamics. No. 1, pp. 91-95, Jan-Mar. 1987.

[31] I.A. Evtushenko, S. Y. Smoletzev. Private Communications. St. Petersburg Technical University in St. Petersburg, USSR. 1991.

[32] C. P. Liao, B. LaBombard, M. S. Kazimi. MHD Equilibrium and Stability of Liquid Metal Film Divertors. Submitted to: Fusion Technology. 1991.

[33] I. A. Evtushenko, S. Y. Smoletzev, A. V. Tananaev. Hydrodynamics and Heat Transfer of Free Surface Flows in a Magnetic Field. To be published in Magnetohydrodynamics. No. 3, Oct-Dec. 1991.

[34] A. Shisko. Private Communication. Latvian Institute of Physics in Riga, Latvia. 1991.

[35] R. L. Panton. Incompressible Flow. John Wiley \& Sons, 1984.

[36] T. N. Aitov, E. M. Kirillina, A. V. Tananaev. Stability of the Flow of a Thin Film of LM in a Coplanar Magnetic Field. Magnetohydodynamics. No. 1, pp. 7-12, Jan-Mar. 1988. 
[37] R. Chodura. Plasma Flow in the Sheath and Pre-Sheath of a Scrape-Off Layer. Physics of Plasma-Wall Interactions in Controlled Fusion. Proceedings of a Nato Advanced Study Institute Series, Plenum Press, pp. 99-134, 1984.

[38] J. A. Shercliff. Theory of Electromagnetic Flow Measurement. Cambridge University Press, 1962.

[39] S. A. Cohen, K. A. Werley, D. E. Post, J. L. Perkins. Two-Dimensional Fluid Simulations of the ITER SOL Plasma. Journal of Nuclear Materials. No. 176-177, pp. 909-915, 1990.

[40] B. D. Nichols, C. W. Hirt, R. S. Hotchkiss. SOLA-VOF: A Solution for Transient Fluid Flow with Multiple Free Boundaries. Los Alamos Scientific Laboratory Report: LA-8355. 1980.

[41] Indium Corperation of America. Indalloy Specialty Solders and Alloys. Utica, NY. 1988.

[42] R. Lyon editor. Liquid-Metals Handbook. Atomic Energy Commision, 1952.

[43] S. Oshima, R. Yamane. Nonlinear Waves of Liquid Metals under a Transverse Magnetic Field. Liquid Metal Flows: MHD Applications. Progress in Astronautics and Aeronautics Series, V. 111, pp. 100-118, 1987.

[44] L. Lamport. A Document Preparation System: $\mathrm{IAT}_{\mathrm{E}} \mathrm{X}$. Addison-Wesley Publising Co. 1986. 


\title{
Appendix A
}

\section{List of symbols and}

\section{abbreviations}

\author{
A ampere (unit of electric current) \\ $b \quad$ channel width $(m)$ \\ $\vec{B} \quad$ magnetic field $(T)$ \\ c specific heat $\left(\frac{\mathrm{J}}{\mathrm{kg}{ }^{\circ} \mathrm{K}}\right)$ \\ C celsius (unit of temperature) \\ $\mathrm{D} / \mathrm{T} \quad$ Deuterium/Tritium \\ DF Droplet Former \\ $\vec{E} \quad$ Electric field $\left(\frac{l^{\prime}}{m}\right)$ \\ $E_{\text {ieio }} \quad$ element of rank-4 (Old) MacDonald tensor \\ $\mathrm{eV}$ electron-Volt (unit of energy, $1 \mathrm{eV}=1.602 \times 10^{-19} \mathrm{~J}$ ) \\ FFX Film Flow eXperiment \\ Fr Froude number $\left(\frac{\left(u_{\circ}\right)^{2}}{g(b / 2)}\right)$
}


$\vec{g} \quad$ acceleration of gravity vector $\left(\mathrm{m} / \mathrm{s}^{2}\right)$

$\mathrm{Ga} \quad$ Gallium

$h \quad$ film height $(m)$

$\mathrm{H}$ Hydrogen, or henries (unit of inductance)

$H a$ Hartmann number $\left(B_{z}^{(0)}(b / 2) \sqrt{\sigma / \rho \nu}\right)$

He Helium

ITER International Thermonuclear Experimental Reactor

$\vec{J} \quad$ current density $\left(\frac{A}{m^{2}}\right)$

J Joules (unit of energy)

Li Lithium

$\mathrm{K} \quad$ Kelvin (unit of temperature)

$k$ thermal conductivity $\left(\frac{\mathrm{W}^{\circ}}{\mathrm{m}^{\circ} \mathrm{K}}\right)$

LM Liquid Metal

m meter (unit of length)

MEGA MEtal Goes Around

MHD MagnetoHydroDynamics

NBM Neil B. Morley

$\vec{n} \quad$ unit normal to free surface $(0, \zeta, \eta)$, or channel walls

$\mathrm{N}$ newton (unit of force)

$p \quad$ pressure $(P a)$

$\mathrm{Pa} \quad$ pascal (unit of pressure)

$Q_{o} \quad$ volumetric flowrate $\left(\mathrm{m}^{3} / \mathrm{s}\right)$

$\vec{R} \quad$ surface stress $\left(N / m^{2}\right)$

Re Reynold's number $\left(\frac{u_{o}(b / 2)}{\nu}\right)$

$R e_{m} \quad$ Magnetic Reynold's number $\left(u_{o} \sigma \mu_{m}(b / 2)\right)$ 


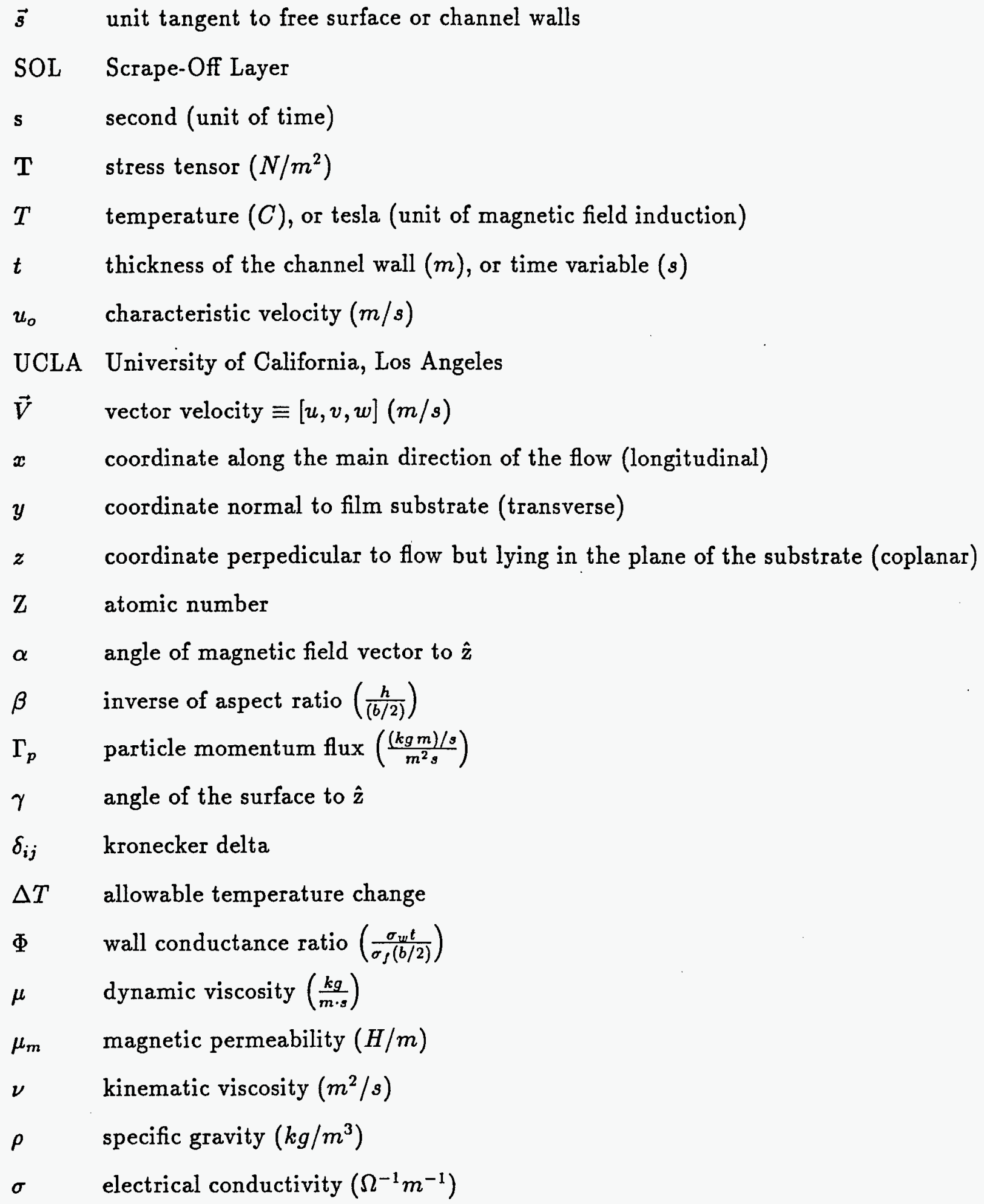


$\tau \quad$ viscous stress tensor $\left(\mathrm{N} / \mathrm{m}^{2}\right)$

$\Omega \quad$ ohm (unit of electrical resistance) 
M97053592

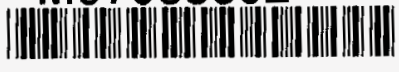

Report Number (14) DOE/OR/O0033--TT28

Publ. Date (11) 1991

Sponsor Code (18) DOE/ER, XF

UC Category (19) UC-400, DOE/ER

DOE 